\title{
Dissecting the spiral galaxy M 83: mid-infrared emission and comparison with other tracers of star formation ${ }^{\star}$
}

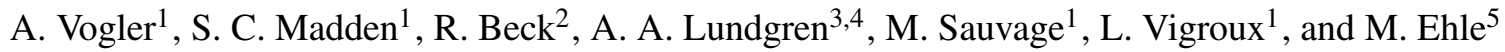 \\ ${ }^{1}$ DSM/DAPNIA/Service d'Astrophysique, CEA/Saclay, 91191 Gif-sur-Yvette Cedex, France \\ e-mail: smadden@cea.fr \\ 2 Max-Planck-Institut für Radioastronomie, Auf dem Hügel 69, 53121 Bonn, Germany \\ 3 European Southern Observatory, Casilla 19001, Santiago 19, Chile \\ ${ }^{4}$ Stockholm Observatory, AlbaNova, 10691 Stockholm, Sweden \\ 5 XMM-Newton Science Operations Centre, European Space Agency, Villafranca, PO Box 50727, 28080 Madrid, Spain
}

Received 9 November 2004 / Accepted 7 July 2005

\begin{abstract}
We present a detailed mid-infrared study of the nearby, face-on spiral galaxy M 83 based on ISOCAM data. M 83 is a unique case study, since a wide variety of MIR broad-band filters as well as spectra, covering the wavelength range of 4 to $18 \mu \mathrm{m}$, were observed and are presented here. Emission maxima trace the nuclear and bulge area, star-formation regions at the end of the bar, as well as the inner spiral arms. The fainter outer spiral arms and interarm regions are also evident in the MIR map. Spectral imaging of the central $3^{\prime} \times 3^{\prime}(4 \mathrm{kpc} \times 4 \mathrm{kpc})$ field allows us to investigate five regions of different environments. The various MIR components (very small grains, polycyclic aromatic hydrocarbon (PAH) molecules, ionic lines) are analyzed for different regions throughout the galaxy. In the total $\lambda 4 \mu \mathrm{m}$ to $18 \mu \mathrm{m}$ wavelength range, the PAHs dominate the luminosity, contributing between $60 \%$ in the nuclear and bulge regions and $90 \%$ in the less active, interarm regions. Throughout the galaxy, the underlying continuum emission from the small grains is always a smaller contribution in the total MIR wavelength regime, peaking in the nuclear and bulge components. The implications of using broad-band filters only to characterize the mid-infrared emission of galaxies, a commonly used ISOCAM observation mode, are discussed. We present the first quantitative analysis of new $\mathrm{H} \alpha$ and $\lambda 6 \mathrm{~cm}$ VLA+Effelsberg radio continuum maps of M 83. The distribution of the MIR emission is compared with that of the $\mathrm{CO}, \mathrm{HI}, R$ band, $\mathrm{H} \alpha$ and $\lambda 6 \mathrm{~cm}$ radio. A striking correlation is found between the intensities in the two mid-infrared filter bands and the $\lambda 6 \mathrm{~cm}$ radio continuum. To explain the tight mid-infrared-radio correlation we propose the anchoring of magnetic field lines in the photoionized shells of gas clouds.
\end{abstract}

Key words. galaxies: individual: M 83 - galaxies: spiral - galaxies: ISM - ISM: dust, extinction - ISM: magnetic fields

\section{Introduction}

Galaxies are composed of stars, gas, dust, dark matter, magnetic fields and cosmic rays, with each component responding differently to local and large-scale dynamical effects. A complete and self-consistent galaxy model should incorporate information on the stellar distribution and its relationship to the atomic, molecular and ionized gas and dust and the subsequent interplay between these ingredients, in order to understand the physics controlling the heating, cooling and star-formation processes. These effects will vary widely from galaxy to galaxy on global scales, as well as within a galaxy between the nucleus, the bar, spiral arms, interarm regions, and outer disk regions. We explore various ISM and star formation properties of the well-studied nearby barred spiral galaxy, M 83, using ISOCAM

^ Based on observations with ISO, an ESA project with instruments funded by ESA Member States (especially the PI countries: France, Germany, the Netherlands and the UK) and with the participation of ISAS and NASA. high-resolution mid-infrared (MIR) spectrophotometric and broad-band observations and a high-resolution $\lambda 6 \mathrm{~cm}$ radio continuum map combined from VLA and Effelsberg observations. We compare our data with results from $\mathrm{H} \alpha$, optical, $\mathrm{CO}$, $\mathrm{HI}$ and X-ray observations.

M 83 is the most prominent spiral galaxy in the nearby Hydra-Centaurus group of galaxies and is one of the largest barred systems in the sky. Due to its proximity ( $4.5 \mathrm{Mpc}$, Thim et al. 2003), its near face-on orientation, and relatively low Galactic foreground absorption $\left(N_{\mathrm{H}} \simeq 4 \times 10^{20} \mathrm{~cm}^{-2}\right.$, Dickey \& Lockman 1990; $E(B-V)=0.066$; Schlegel et al. 1998), it is ideally suited for the study of morphological and physical variations of the ISM. M 83 has been observed extensively at different energies, ranging from the long-wavelength radio to the X-ray bands. A rather large data set has been accumulated including atomic gas (Huchtmeier \& Bohnenstengel 1981; Tilanus \& Allen 1993), H $\alpha$ (Ryder et al. 1995; Lundgren et al. 2005), the molecular gas (Handa et al. 1990; Petitpas \& Wilson 1998; Crosthwaite et al. 2002; Lundgren et al. 2004a,b; 
Table 1. ISOCAM observations of M 83. TDT is the Target Dedicated Time Number of the observation.

\begin{tabular}{ccccccc}
\hline \hline Filter & $\begin{array}{c}\text { Band } \\
(\mu \mathrm{m})\end{array}$ & Date & $\begin{array}{c}\text { Mean exposure } \\
(\mathrm{s})\end{array}$ & $\begin{array}{c}\text { Raster } \\
\text { mode }\end{array}$ & $\begin{array}{c}\text { Field size } \\
(\mathrm{kpc})\end{array}$ & $\begin{array}{c}\text { TDT } \\
\text { number }\end{array}$ \\
\hline LW1 & $4.00-5.00$ & 16 Aug. 1997 & 243 & $2 \times 9$ & $23 \times 7$ & 63900451 \\
LW2 & $5.00-8.50$ & 23 Aug. 1997 & 224 & $7 \times 7$ & $17 \times 17$ & 64700246 \\
LW3 & $12.0-18.0$ & 23 Aug. 1997 & 224 & $7 \times 7$ & $17 \times 17$ & 64700350 \\
LW4 & $5.50-6.50$ & 16 Aug. 1997 & 243 & $2 \times 9$ & $23 \times 7$ & 63900451 \\
$L W 5$ & $6.50-7.00$ & 16 Aug. 1997 & 243 & $2 \times 9$ & $23 \times 7$ & 63900451 \\
$L W 6$ & $7.00-8.50$ & 08 Aug. 1997 & 234 & $2 \times 9$ & $23 \times 7$ & 63200147 \\
$L W 7$ & $8.50-10.7$ & 08 Aug. 1997 & 234 & $2 \times 9$ & $23 \times 7$ & 63200147 \\
$L W 8$ & $10.7-12.0$ & 08 Aug. 1997 & 234 & $2 \times 9$ & $23 \times 7$ & 63200147 \\
$L W 9$ & $14.0-16.0$ & 08 Aug. 1997 & 234 & $2 \times 9$ & $23 \times 7$ & 63200147 \\
CVF & $5.00-16.5$ & 24 Aug. 1997 & & single obs. & $4 \times 4$ & 64800263 \\
\hline
\end{tabular}

Sakamoto et al. 2004) and X-rays (Trinchieri et al. 1985; Ohashi et al. 1990; Okada et al. 1997; Ehle et al. 1998; Immler et al. 1999; Soria \& Wu 2002; 2003). M 83 is also bright in radio continuum due to its high star-formation rate and strong magnetic field. Highly polarized emission found in M 83 suggests a regular magnetic field with a spiral pattern (Neininger et al. 1991; 1993).

This study was motivated by the rich assortment of MIR ISO data on M 83. By now, almost 10 years after the launch of ISO, we are beginning to understand the utility of the various tracers in the MIR wavelength domain.

This paper is organized as follows: Sect. 2 presents the observational details, Sect. 3 the decomposition and description of the MIR spectral signatures in various selected regions within M 83. The spectral information is then used to decipher the broad-band observations. This is an important demonstration of what is actually being traced by the ISOCAM broadbands; simple interpretations of the broad-bands do not apply everywhere in a galaxy. We also explore the ratios of the mid-infrared PAH bands throughout the galaxy. Section 4 presents the radio continuum interpretation of M 83. Section 5 presents the MIR observations in light of $\mathrm{H} \alpha$, X-ray emission and molecular gas. In Sect. 6 we discuss the spatial distributions of the various tracers and zoom into the bar, the nuclear region and the south-eastern spiral arm. In Sect. 7, we quantify the correlations between the intensities of the MIR bands and the other ISM components presented here and discuss the utility of the MIR characteristics as a tracer of star formation.

\section{Observations and data analysis}

Observation logs and relevant instrument features for the midinfrared (MIR) and radio continuum observations are presented in Table 1 and Sect. 2.3, respectively. For some of the published images, we present overlays of our data on the published images (Figs. 7-10, 12, 13). References of the published data used for analysis purposes in this paper, along with instrumental features, are given in Table 2.

\subsection{ISOCAM images}

The MIR data were obtained with the Infrared Space Observatory (ISO; Kessler et al. 1996) using the ISOCAM instrument (Cesarsky et al. 1996). This instrument provided imaging modes with a variety of broad-band filters from $\lambda 4 \mu \mathrm{m}$ to $18 \mu \mathrm{m}(L W 1-L W 9)$ and a spectral imaging mode, using a circular variable filter (CVF) from $\lambda 5 \mu \mathrm{m}$ to $16.5 \mu \mathrm{m}$. Table 1 displays more details of the various filters used for the data presented here. The ISOCAM detector has a $32 \times 32$ pixel field of view, and here one pixel corresponds to $6^{\prime \prime} \times 6^{\prime \prime}$ $(130 \mathrm{pc} \times 130 \mathrm{pc})$ on the sky. The full-width-at-half-maximum $(F W H M)$ of the diameters of the point spread functions (PSF) with a $6^{\prime \prime}$ pixel size range from $5^{\prime \prime} .8$ at $7 \mu \mathrm{m}$ to $8^{\prime \prime}$ for $\lambda 15 \mu \mathrm{m}$.

The $L W 2$ and $L W 3$ broad-band filters, centered at $\lambda 6.7 \mu \mathrm{m}$ and $\lambda 15 \mu \mathrm{m}$, respectively, were used to observe $7 \times 7$ raster maps, which contain the full galaxy plus sky background. The images overlapped by half of an array size, shifted by $1 / 2$ of a pixel, and were combined to obtain a total image covering

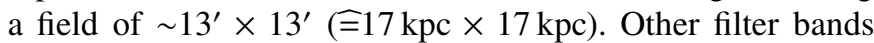
were observed in a $2 \times 9$ raster mode, covering $17^{\prime} \times 5.4^{\prime}$ $(\widehat{=} 23 \mathrm{kpc} \times 7 \mathrm{kpc}$ ) fields centered on the nucleus of M 83 (see Table 1). Given the optical extent of the galaxy of $12: 9 \times 11.5$ $(\widehat{=} 17 \mathrm{kpc} \times 15 \mathrm{kpc})$, determination of the field background is somewhat limited for the $7 \times 7$ raster maps, while it is more straight forward for the $2 \times 9$ raster maps. Thus, we determined the background from regions of our map outside the optical extent of M 83, located between 7'.5 and $9^{\prime}$ from the center. Subtracting these backgrounds, M 83 is detected with a total MIR flux of $20 \mathrm{Jy}$ and $21 \mathrm{Jy}$ in the $L W 2(\lambda 6.75 \mu \mathrm{m})$ and $L W 3(\lambda 15.0 \mu \mathrm{m})$ band, respectively. Typical errors are of the order of $15 \%$, and our fluxes compare well to those of Roussel et al. (2001b), as well as with the IRAS $12 \mu \mathrm{m}$ flux of $26 \mathrm{Jy}$ with an error of the same order as our MIR values.

The filters used for the strip maps include a variety of narrow-bands within the broader wavelength domain of the $L W 2$ and $L W 3$ bands (cf. Table 1 and the sketched bandwidths in Figs. 3 and 4). The narrow-band filters allow us to interpret the MIR contributions with better accuracy. While not covering the complete optical diameter of the galaxy, the strip 
Table 2. References to further M 83 images used in this study.

\begin{tabular}{llcl}
\hline \hline & Field size & FWHM & Reference \\
& or field of view (FOV) & $\left({ }^{\prime \prime}\right)$ & \\
\hline Calibrated H $\alpha$ & $12^{\prime} \times 12^{\prime}$ & 1.1 & Lundgren et al. (2005) \\
H I & FOV $\simeq 30^{\prime}$ & 12 & Tilanus \& Allen (1993) \\
CO $(J=1-0)$ & South-eastern spiral arm & $6.5 \times 3.5$ & Rand et al. (1999) \\
CO $(J=1-0)$ & Nuclear region and bar ${ }^{1}$ & 16 & Handa et al. (1990) \\
CO $(J=1-0)$ & $10^{\prime} \times 10^{\prime}$ & 22 & Lundgren et al. (2004a) \\
CO $(J=2-1)$ & $10^{\prime} \times 10^{\prime}$ & 14 & Lundgren et al. (2004a) \\
CO $(J=3-2)$ & Nuclear region & 14 & Petitpas \& Wilson (1998) \\
X-rays, ROSAT PSPC & FOV $\simeq 2^{\circ}$ & $25-52$ & Ehle et al. (1998) \\
Radio continuum $(\lambda 6 \mathrm{~cm})$ & $40^{\prime} \times 40^{\prime}$ & 12 & This paper \\
\hline
\end{tabular}

${ }^{1}$ And additional points with 7'.5 stepping close to the nucleus.

maps of M 83 with scan direction from the north-east to the south-west $(17 ! 4 \times 5 ! 4)$, offer the advantage of determining the field background more precisely. The background values for the strip maps were extracted from 2 regions of $3^{\prime} \times 2^{\prime}$ (long axis perpendicular to major axis of the strip) beyond the optical extent of the galaxy. We verified the $L W 2$ and $L W 3$ background determination from the $7 \times 7$ raster maps, using the variety of strip maps.

The data analysis was performed within the CEA/CIR (Chanial \& Gastaud 2000) and ESO/MIDAS software environments.

Most cosmic ray impacts, resulting in short duration glitches, were removed using a multi-resolution median filtering technique in CIR, while, for the non-classical longer duration glitches, more sophisticated secondary techniques described in Roussel et al. (2001a) were employed. Transient effects, due to long term memory effects of the detectors before stabilization is achieved, were corrected using the FouksSchubert method (Coulais \& Abergel 2000). The flat-field correction was computed by masking the source and computing the median of the remaining field. See Roussel et al. (2001a) for more specific details.

Figures $1 \mathrm{a}$ and $\mathrm{b}$ show the entire galaxy in the MIR light centered at $\lambda 6.75 \mu \mathrm{m}$ ( $L W 2$; resolution $\left.5^{\prime \prime} .7\right)$ and $\lambda 15.0 \mu \mathrm{m}$ (LW3; resolution 6.' 1 ).

Since the broad-band images can contain emission from various dust components, molecules, ionic species, etc. (see Sect. 3.1), spectro-imaging data were also obtained with a single array setting of the central $3 ! 2 \times 3 ! 2(\widehat{=} 4 \mathrm{kpc} \times 4 \mathrm{kpc}$ ) region of M 83 to facilitate the interpretation of the broad band observations. The spectral resolution of the CVF was 40 . Transient correction, deglitching and flat fielding were performed, basically, as for the broad-band observations described above. Since the array was never off the galaxy completely, background subtraction was not done in the usual manner as described above for photometric mapping observations. Rather, the maximum and minimum zodiacal light spectra were estimated and subtracted from the galaxy spectrum, providing limits to the resulting spectrum. This procedure is described in detail in Roussel et al. (2001a).

\subsection{Astrometry corrections of the ISOCAM observations}

To test the position accuracy of the MIR data, we searched for optical counterparts of the MIR sources outside the optical extent of M 83 in the Digitized Sky Survey-2 (DSS-2) image of the M 83 field (from the ESO online archive). For the LW2 image, we found counterparts for five MIR sources (three foreground stars, two background galaxies). A transformation matrix from the proposed MIR astrometry to the astrometry implied by the optical image was calculated. A translation of $3^{\prime \prime}$ to the west and $4^{\prime \prime}$ to the south, and, in addition, a clockwise rotation of 1.4 was proposed. We corrected the $L W 2$ image for these values achieving a position accuracy of $\sim 2$.' 5 , and then used the LW2 image when overlaying MIR data on images at other wavelengths.

An overlay of the rotated and shifted $L W 2$ data on the DSS image of M 83 is presented in Fig. 2.

\subsection{Radio continuum}

M 83 was observed at $4.885 \mathrm{GHz}(\lambda 6.14 \mathrm{~cm})$ with the VLA synthesis radio telescopes by $\mathrm{NRAO}^{1}$ in its $\mathrm{DnC}$ configuration on 1992 June 27 and 28 by S. Sukumar. To cover most of the galaxy, two fields were observed, centered at $\mathrm{RA}_{2000}=13^{\mathrm{h}} 36^{\mathrm{m}} 54^{\mathrm{s}}, \operatorname{Dec}_{2000}=-29^{\circ} 49^{\prime} 31^{\prime \prime}$ (north-west) and $\mathrm{RA}_{2000}=13^{\mathrm{h}} 37^{\mathrm{m}} 09^{\mathrm{s}}, \mathrm{Dec}_{2000}=-29^{\circ} 54^{\prime} 06^{\prime \prime}$ (southeast). Maps of the two fields were generated with a synthesized beam of $10^{\prime \prime}$ half-power beam width, corrected for primarybeam attenuation and combined into one map. The rms noise is $\simeq 20 \mu \mathrm{Jy}$ per beam over the region of the main spiral arms, but increases towards the edges of the map. As the VLA map does not include any structures of more than about $5^{\prime}$ extent, most of the diffuse radio emission is not detected.

\footnotetext{
1 The National Radio Astronomy Observatory is a facility of the National Science Foundation operated under cooperative agreement by Associated Universities, Inc.
} 

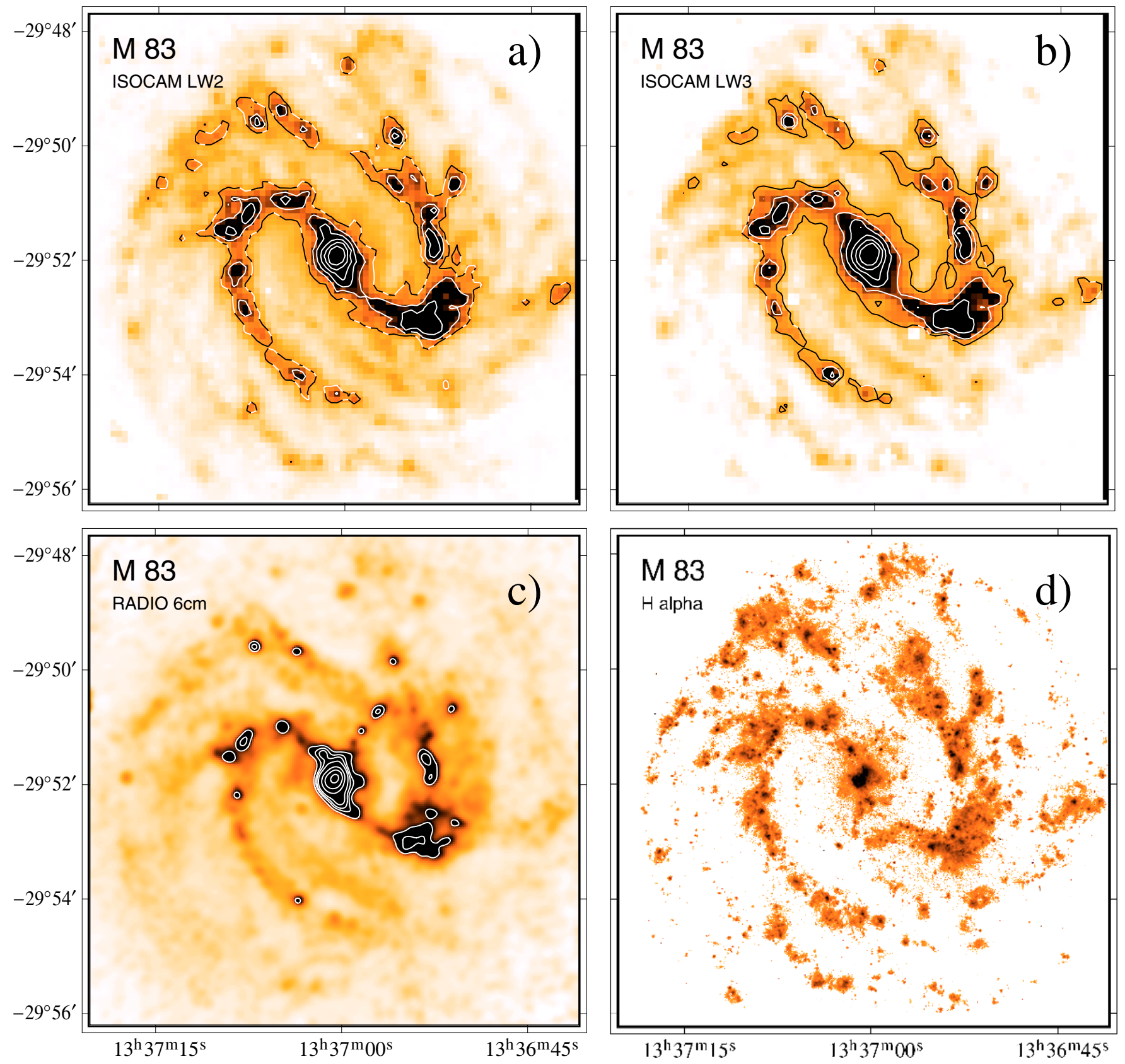

Fig. 1. a) and b) ISOCAM $L W 2$ and $L W 3$ images of M 83 (resolution of 5". 8 and 8", respectively). The dynamical range of the gray-scale intensity representation runs from 0.5 to $\sim 5 \mathrm{mJy}$ per image pixel $\left(36 \mathrm{arcsec}^{2}\right)$ above the background $(8.15 \mathrm{mJy}$ per image pixel for the $L W 2$ band, $39.3 \mathrm{mJy}$ per image pixel for the $L W 3$ band), regions of higher intensity have been sketched with contours, namely at levels of $5,9,15,30,60$ and $120 \mathrm{mJy}$ above the background per image pixel. c) $\lambda 6 \mathrm{~cm}$ combined Effelsberg and VLA radio image. Contour levels are 2, 3, 4, 5, 10, 30, $50 \mathrm{mJy} /$ beam (angular resolution 12"). d) H $\alpha$ image of M 83. The continuum-contribution has been subtracted away using narrow-band data. These observations were carried out with the Danish $1.54 \mathrm{~m}$ telescope and DFOSC at ESO, La Silla, Chile. See Lundgren et al. (2005) for more details.

A field of $40^{\prime} \times 40^{\prime}$ encompassing M 83 was observed in 1996 with the Effelsberg 100-m telescope ${ }^{2}$ using the $4.85 \mathrm{GHz}$ receiver system providing an angular resolution of 2.6. The final map has an rms noise of $\simeq 1 \mathrm{mJy}$ per beam. Emission from M 83 is detected out to about $6^{\prime}(\widehat{=} 8 \mathrm{kpc}$ ) radius.

\footnotetext{
${ }^{2}$ The 100-m Effelsberg telescope is operated by the Max-Planck-Institut für Radioastronomie on behalf of the Max-Planck-Gesellschaft (MPG).
}

The VLA and Effelsberg data were merged in the Fourier plane using IMERG based on NRAO's SDE software (see Beck \& Hoernes 1996). The final map has the same angular resolution and rms noise as the original VLA map, but includes all diffuse extended emission (about $35 \%$ of the total flux) detected with the Effelsberg single-dish. The map is shown in Fig. 1c. The polarization map was presented by Beck (2002) and will be discussed in a future paper (Beck et al. 2005). 


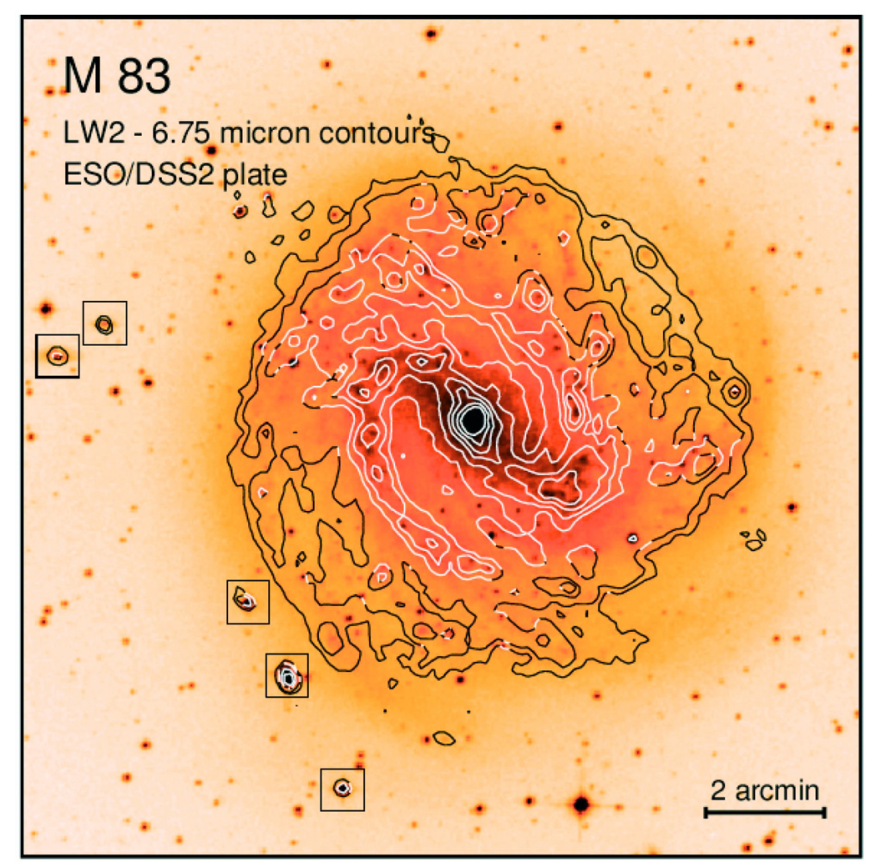

Fig. 2. ISOCAM $L W 2(\lambda 6.75 \mu \mathrm{m}$ central wavelength; angular resolution $6^{\prime \prime}$ ) contours on the DSS-2 image from the ESO archive. Five background galaxies detected outside of M 83 were used for astrometry and are outlined by boxes. After alignment of the background galaxies with their optical counterparts, the error of the astrometry solution is $\leq 2$ ". 5 .

\section{Results}

\subsection{The ISOCAM MIR View of M 83}

The ISOCAM CVF wavelengths ranges from $\lambda 5 \mu \mathrm{m}$ to $17 \mu \mathrm{m}$ and traces a variety of dust components as well as ionic and molecular gas lines, depending on local environmental conditions within galaxies. The MIR dust components primarily consist of 2 types in galaxies: (1) small particles or large molecules (e.g., polycyclic aromatic hydrocarbon molecules; $\mathrm{PAHs}^{3}$ ), stochastically heated, undergoing large temperature fluctuations of the order of hundreds of K on short timescales as a result of single photon heating processes (e.g. Puget \& Leger 1984; Sellgren et al. 1990; Allamandola et al. 1989; Verstraete et al. 2001; Krügel 2003), with major emission bands primarily at $\lambda 3.3 \mu \mathrm{m}, 6.2 \mu \mathrm{m}, 7.7 \mu \mathrm{m}, 8.6 \mu \mathrm{m}, 11.3 \mu \mathrm{m}$ and $12.7 \mu \mathrm{m}$; (2) very small grains (VSGs) which typically have sizes in our Galaxy in the range of $1 \mathrm{~nm}-150 \mathrm{~nm}$ (Désert et al. 1990) and which are the origin of the MIR continuum in our ISOCAM spectra, longward of $\lambda 11 \mu \mathrm{m}$. These VSGs, representing a range of temperatures, could be either stochastically heated or in thermal equilibrium, depending on the radiation field properties and grain sizes. Both PAHs and VSGs are excited mostly via UV photons in the vicinity of active star formation regions. The PAHs are observed to peak in the photodissociation regions (PDRs), which are the interface regions between molecular cores and HII regions. The VSGs emit prominently in the nebular regions (e.g. Cesarsky et al. 1996;

\footnotetext{
3 Although the precise nature of these particles is uncertain, we will refer to these as PAH bands throughout the paper.
}
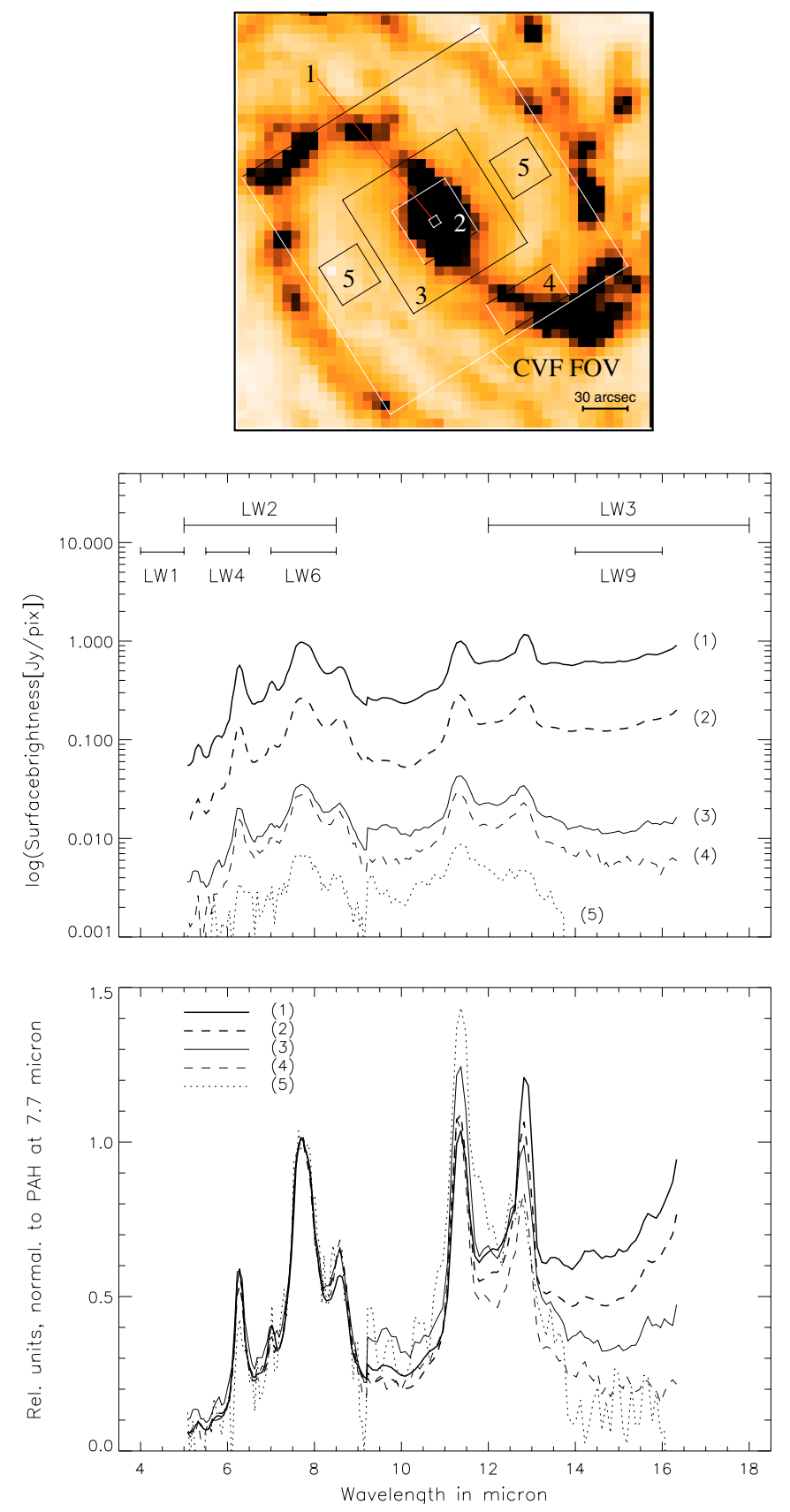

Fig. 3. Top panel: the boxes mark the ISOCAM CVF field of view and extracted regions for spectra on top of the ISOCAM $L W 2(\lambda 6.75 \mu \mathrm{m}$ central wavelength) image. Middle panel: CVF spectra for five different regions, namely (1) the central pixel of the M 83 field; (2) the $7 \times 7$ central pixels excluding the region (1); (3) the central $11 \times 11$ pixels excluding regions (1) and (2); (4) a $9 \times 4$ pixel on-arm region offset from the bulge; (5) average of two $5 \times 5$ pixel interarm regions. Bottom panel: the same spectra normalized to the $\lambda 7.7 \mu \mathrm{m}$ PAH emission and on a linear scale.

Verstraete et al. 1996). On the other hand, PAHs can also be excited by less energetic means, such as optical photons, as evidenced by their presence in the diffuse interstellar medium (ISM) (Chan et al. 2001) and elliptical galaxies (e.g. Athey et al. 2002; Xilouris et al. 2004).

In the case of more active regions, such as the nuclei of normal galaxies, starbursts or active galactic nuclei, various 

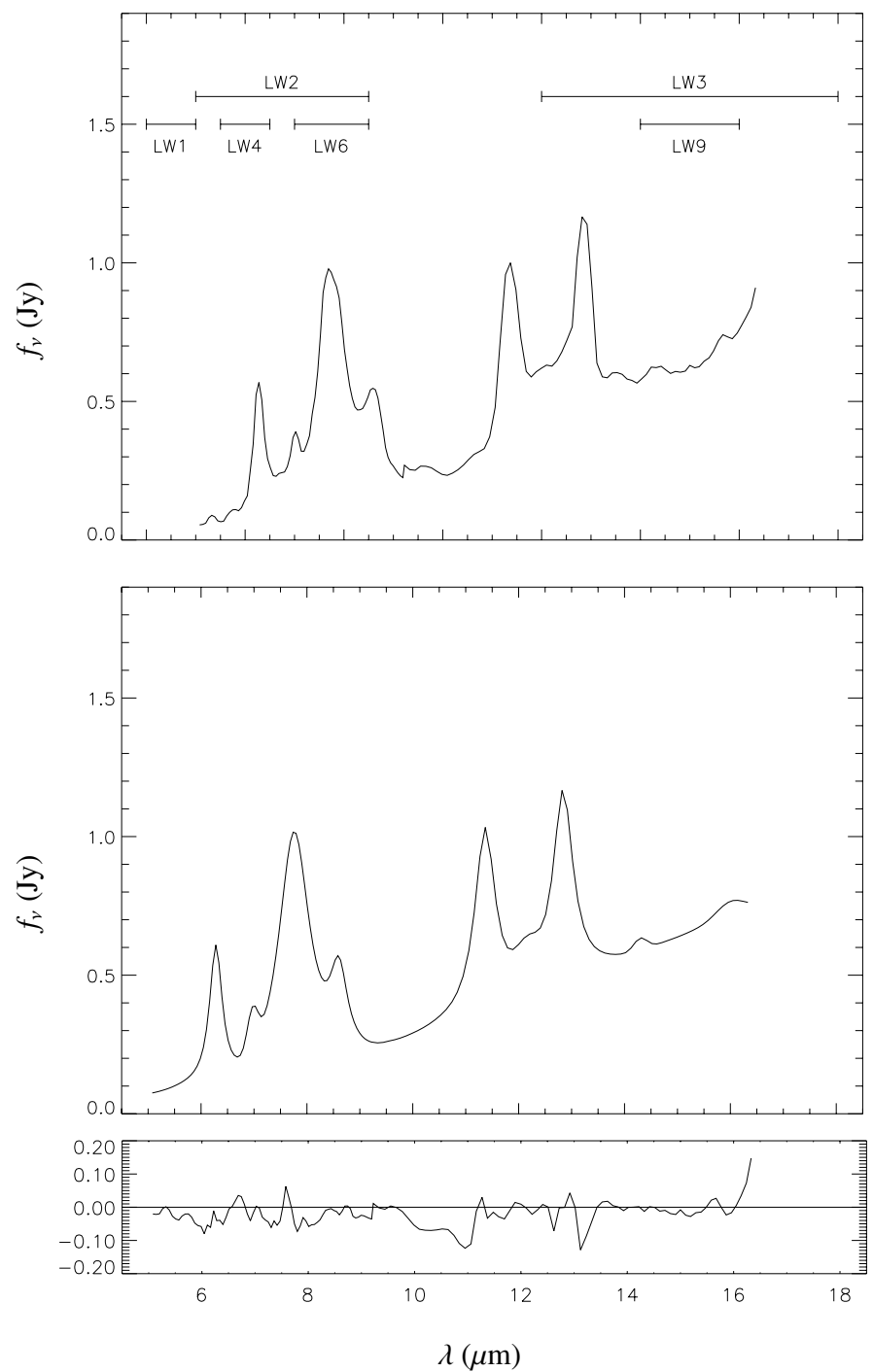

Fig. 4. ISOCAM MIR spectrum of the central pixel of the M 83 observation. The $x$-axis gives the wavelength in $\mu \mathrm{m}$, the $y$-axis the flux in Jy at the spectral resolution of the ISOCAM CVF. Top panel: observed spectrum. The $5 \mathrm{PAH}$ bands figure prominently at $6.2 \mu \mathrm{m}, 7.7 \mu \mathrm{m}$, $8.6 \mu \mathrm{m}, 11.3 \mu \mathrm{m}$ and $12.7 \mu \mathrm{m}$, while the ionic line ([ArII] is also seen at $\lambda 7.0 \mu \mathrm{m}$. The bandwidths of the $L W 1, L W 2, L W 3, L W 4, L W 6$ and $L W 9$ filters are indicated. Middle panel: fitted lines plus continuum. The spectra of all the fitted lines and the continuum were co-added and represented as the modeled spectra. The model details are described in Sect. 3.2. Bottom panel: difference between observed and fitted spectrum.

nebular lines can also be observed in the ISO MIR wavelength range (e.g. Genzel et al. 1998; Rigopoulou et al. 2002; Sturm et al. 2002; Lutz et al. 2003; Förster Schreiber et al. 2003; Verma et al. 2003; Madden et al. 2005). In spiral galaxies (i.e. Roussel et al. 2001a,b) the nuclear region can show a $\lambda 12.8 \mu \mathrm{m}$ [NeII] line, and in harder radiation fields the $\lambda 15.6 \mu \mathrm{m}$ [NeIII] line can be present, too. A more detailed discussion of the general MIR characteristics of spiral galaxies and starbursts is given, e.g., in Vigroux et al. (1999), Dale et al. (2000), Genzel \& Cesarsky (2000) and Krügel (2003).

\subsection{Spectral properties of the nucleus, spiral arms, bulge and interarm regions}

In order to determine the MIR properties of various environments within a typical spiral galaxy, such as M 83, the galaxy is subdivided into the following regions (outlined in Fig. 3) from which we extracted spectra for comparison:

1. The central and brightest $6^{\prime \prime} \times 6^{\prime \prime}(130 \mathrm{pc} \times 130 \mathrm{pc})$ pixel around the nucleus of M 83. This includes much of the starburst arc of the central region of the galaxy (Sakamoto et al. 2004).

2. A $42^{\prime \prime} \times 42^{\prime \prime}(910 \mathrm{pc} \times 910 \mathrm{pc})$ region centered on the nucleus, excluding the innermost pixel and containing the extended circumnuclear environment.

3. A $90^{\prime \prime} \times 90^{\prime \prime}(2 \mathrm{kpc} \times 2 \mathrm{kpc})$ region highlighting the bulge and centered on the nucleus, excluding the nuclear regions (1) and (2).

4. A $24^{\prime \prime} \times 54^{\prime \prime}(520 \mathrm{pc} \times 120 \mathrm{pc})$ region centered on the SW spiral arm and excluding the bulge region.

5. An interarm region containing two $30^{\prime \prime} \times 30^{\prime \prime}(650 \mathrm{pc} \times$ $650 \mathrm{pc}$ ) regions between the bulge and inner spiral arms.

Figure 3 (middle panel) displays the variety of spectra for the different regions. For the spectrum of region (5), the interarm region, the 2 regions on either side of the bulge are averaged to enhance the signal to noise ratio. The most striking features identified in the spectra of Fig. 3 toward all regions are the PAH bands at $\lambda 6.2 \mu \mathrm{m}, 7.7 \mu \mathrm{m}, 8.6 \mu \mathrm{m}$ and $11.3 \mu \mathrm{m}$, and a blended [NeII] and PAH feature at $\simeq \lambda 12.8 \mu \mathrm{m}$. There is a general tendency for the continuum emission from hot grains beyond $\simeq \lambda 13 \mu \mathrm{m}$ to peak toward the nucleus, and to decrease toward the outer, more quiescent regions, as demonstrated in Fig. 3 (bottom panel, where the spectra are normalized at $\lambda 7.7 \mu \mathrm{m}$ ). This was also illustrated for other spiral galaxies in Roussel et al. (2001a) and starburst galaxies (i.e. Förster-Schreiber et al. 2003). The shape and the intensity of the spectra from $\lambda 5 \mu \mathrm{m}$ to $11 \mu \mathrm{m}$, dominated by the cluster of PAH bands, varies less so throughout the galaxy.

For quantitative analysis we decomposed the spectra, fitting them with 3 components (Laurent et al. 2000): 1) PAH bands (the major PAH bands: $\lambda 3.3 \mu \mathrm{m}, 6.2 \mu \mathrm{m}, 7.7 \mu \mathrm{m}, 8.6 \mu \mathrm{m}$, $11.3 \mu \mathrm{m}$ and $12.7 \mu \mathrm{m}$ and the less prominent bands: $\lambda 5.3 \mu \mathrm{m}$, $5.7 \mu \mathrm{m}, 12.0 \mu \mathrm{m}, 13.6 \mu \mathrm{m}$ and $14.3 \mu \mathrm{m})$ were fitted with Lorentzian profiles (i.e. Boulanger et al. 1998); 2) the ionic lines ([ArII] $\lambda 7.0 \mu \mathrm{m}$, [ArIII] $\lambda 9.6 \mu \mathrm{m}$, [SIV] $\lambda 10.5 \mu \mathrm{m}$, [NeII] $\lambda 12.8 \mu \mathrm{m}$ and [NeIII] $\lambda 15.6 \mu \mathrm{m}$ ) were modeled with Gaussian profile characteristics and 3) the continua were assumed to have power-law shapes. The central wavelengths were fixed and the program computed the width and intensity. The index of the continuum power law was a free parameter. The different spectral components of the CVF spectra are illustrated in Fig. 4 for the case of region (1). The figure shows the observed CVF spectrum (above panel), the model lines and bands plus continuum for comparison (middle panel). The very low residual emission (Fig. 4, bottom panel) after the subtraction of the model spectrum from the observation, speaks well for the assumptions in the modeling of the CVF spectra. 
Table 3. Comparison of MIR components using CVF observations of the central $3^{\prime}$ field of M 83. (Note: "Cont." in the table refers to the continuum emission.)

\begin{tabular}{|c|c|c|c|c|c|c|c|c|c|c|}
\hline $\begin{array}{l}\text { Region } \\
\text { (see } \\
\text { Fig. 3) }\end{array}$ & $\begin{array}{c}\text { PAHs } \\
10^{-11} \\
\mathrm{erg} / \mathrm{s} \\
/ \mathrm{cm}^{2}\end{array}$ & $\begin{array}{l}\text { Cont. } \\
10^{-11} \\
\mathrm{erg} / \mathrm{s} \\
/ \mathrm{cm}^{2}\end{array}$ & $\begin{array}{c}\text { PAHs: } \\
\text { total } \\
4-18 \\
\mu \mathrm{m}\end{array}$ & $\begin{array}{c}\text { PAHs: } \\
\text { Cont. } \\
4-18 \\
\mu \mathrm{m}\end{array}$ & $\begin{array}{l}\text { PAHs: } \\
\text { Cont. } \\
L W 2\end{array}$ & $\begin{array}{l}\text { PAHs: } \\
\text { Cont. } \\
L W 3^{1}\end{array}$ & $\begin{array}{r}L W 2 / L W 3 \\
\text { from CVF } \\
6.7 / 15 \mu \mathrm{m}\end{array}$ & $\begin{array}{l}L W 9 / L W 6 \\
\text { from CVF } \\
15 / 7.7 \mu \mathrm{m}\end{array}$ & $\begin{array}{c}\text { PAHs } \\
6.2: 7.7: 8.6: 11.3 \\
\mu \mathrm{m}\end{array}$ & $\begin{array}{c}\text { PAHs } \\
11.3 / 12.8 \\
\mu \mathrm{m}\end{array}$ \\
\hline (1) & 10.7 & 6.6 & 0.62 & 1.6 & 13 & 0.26 & 0.57 & 1.1 & $0.32: 1: 0.16: 0.22$ & 1.25 \\
\hline (2) & 71.3 & 31.5 & 0.69 & 2.3 & 17 & 0.34 & 0.69 & 0.8 & $0.31: 1: 0.22: 0.29$ & 1.49 \\
\hline (3) & 42.0 & 17.7 & 0.70 & 2.4 & 13 & 0.6 & 0.91 & 0.6 & 0.37:1:0.19:0.33 & 1.37 \\
\hline (4) & 3.4 & 1.1 & 0.76 & 3.0 & 20 & 0.7 & 1.22 & 0.4 & 0.29:1:0.26:0.29 & 1.41 \\
\hline (5) & 2.7 & 0.3 & 0.90 & 9.1 & $\geq 100$ & 3.3 & 1.75 & 0.2 & 0.42:1:0.11:0.59 & 2.48 \\
\hline
\end{tabular}

${ }^{1}$ A possible blend of the PAH band with the [NeII] line at $\simeq \lambda 12.8 \mu \mathrm{m}$ cannot be resolved by the CVF. Typical contributions to the [NeII] line are expected to be small (e.g. Roussel et al. 2001a; Sturm et al. 2000), and are assumed to be below 30\%, with the remainder attributed to the $\mathrm{PAH}$ feature.

The model fits are used for quantitative comparisons in the following discussion.

Typical flux densities for the brightest PAH features are $\gtrsim 0.5 \mathrm{mJy}$, while atomic line contributions are smaller, the largest being the [NeII] $\lambda 12.8 \mu \mathrm{m}$ line $(0.2 \mathrm{mJy})$ towards the nucleus. We detect minor contributions from the [ArII] $7.0 \mu \mathrm{m}$ line. The [NeIII] $15.6 \mu \mathrm{m}$ line appears to be present in the nucleus (regions 1 and 2) and the bulge (region 3 ) and not evident in regions 4 (on arm) and 5 (interarm). Throughout the galaxy, PAHs seem to be ubiquitous and account for the majority of the MIR 4 to $18 \mu \mathrm{m}$ luminosity (Table 3). The continuum emission is at most $40 \%$ of the total MIR emission toward the central starburst core (region 1), and decreases away from the central region. Toward the interarm region (region 5), the PAHs account for almost all of the MIR energy. Both the $L W 2$ and $L W 3$ images (Sect. 3.5), as well as the CVF spectra, show MIR emission in the form of PAH bands but no significant continuum towards the interarm or outer regions. Toward diffuse regions of the Galaxy, the PAH bands are the fundamental component of the MIR emission (e.g. Mattila et al. 1996; Lemke et al. 1998; Chan et al. 2001).

\subsection{Using the CVF to determine the broad-band composition}

The ISOCAM LW2 $(\lambda 5.0-8.5 \mu \mathrm{m})$ and $L W 3$ $(\lambda 12.0-18.0 \mu \mathrm{m})$ bands were popular broad-bands observed with ISO and often were the only observations used to characterize the MIR ISM of galaxies. Depending on the type of galaxy, these bands encompass various components to varying degrees. Often it was simply assumed that the $L W 2$ band is dominated by PAH bands, while the $L W 3$ traces the dust continuum. Here we take advantage of the fact that a variety of broad-bands as well as CVF spectra were obtained for M 83 to quantify the contribution of the various MIR components to each of these broad-bands, using the CVF spectra. We can then interpret the ISOCAM MIR broad-band $L W 2 / L W 3$ ratio in galaxies more accurately.

In Table 3 we present the results for the modeled CVF for the different regions in M 83 (refer to Fig. 3 for definitions of the region numbers) extracting continua and PAH band contributions. The cluster of PAH bands at $\lambda 8 \mu \mathrm{m}(\lambda 6.2,7.7,8.6 \mu \mathrm{m})$ is always dominant in the $L W 2$ broad-band filter throughout M 83, with the continua being on the order of about $5 \%$ of the total $L W 2$ broad-band flux. We do not detect any substantial contribution from the very hot grain continua or stellar emission from evolved populations, emitting below $\lambda 9 \mu \mathrm{m}$ in the CVF spectra. Hot grain continuum emission at these shorter MIR wavelengths has been detected in active galactic nuclei and prominent starbursts (Hunt et al. 2002; Madden et al. 2005). In early-type galaxies, this spectral range is dominated by stellar emission (e.g. Xilouris et al. 2004). A substantial fraction of the flux in the $L W 3$ band arises from the continuum emission: $75 \%$ to $80 \%$ in the central region, $\simeq 60 \%$ in the bulge and spiral arms. In the more quiescent interarm regions, however, the $\lambda 12.7 \mu \mathrm{m}$ PAH band dominates the $L W 3$ band emission $(\simeq 75 \%)$.

While it is tempting to use the $L W 2 / L W 3$ ratio to study the MIR emission in galaxies, given the different emission features contributing to the broad-band filters, it would be preferable to choose a filter isolating a PAH band only, and another which selects only the continuum of $\lambda>10 \mu \mathrm{m}$. The $L W 6$ broadband filter $(\lambda 7.0 \mu \mathrm{m}$ to $8.5 \mu \mathrm{m})$, for example, only traces the $\lambda 7.7 \mu \mathrm{m}$ PAH band and part of the $\lambda 8.6 \mu \mathrm{m}$ PAH band while the $L W 9(\lambda 14.0 \mu \mathrm{m}$ to $\lambda 16.0 \mu \mathrm{m})$ filter traces the $\lambda 15 \mu \mathrm{m}$ continuum (when the [Ne III] line is not present, as is the case here), removing possible ambiguities (Fig. 4). The CVF predicts a ratio of $L W 9 / L W 6(\lambda 15 \mu \mathrm{m}$ continuum $) /(\lambda 7.7 \mu \mathrm{m}$ PAH $)$ of 1.1 for the nuclear region and decreases to 0.2 for the diffuse interarm region. In M 83 the dynamical range of the LW9/LW6 intensity ratio (factor of 5, see Fig. 5) is larger than that of the $L W 2 / L W 3$ ratio (factor of 2, see Sect. 3.5), making it a more sensitive tool to distinguish different physical regions of a galaxy.

\section{4. $P A H$ band ratios in $M 83$}

The ratios of the PAH band intensities are a means of testing the validity of the PAH model assumption for the $\lambda 6.2 \mu \mathrm{m}, 7.7 \mu \mathrm{m}, 8.6 \mu \mathrm{m}$ and $11.3 \mu \mathrm{m}$ and $12.7 \mu \mathrm{m}$ bands 


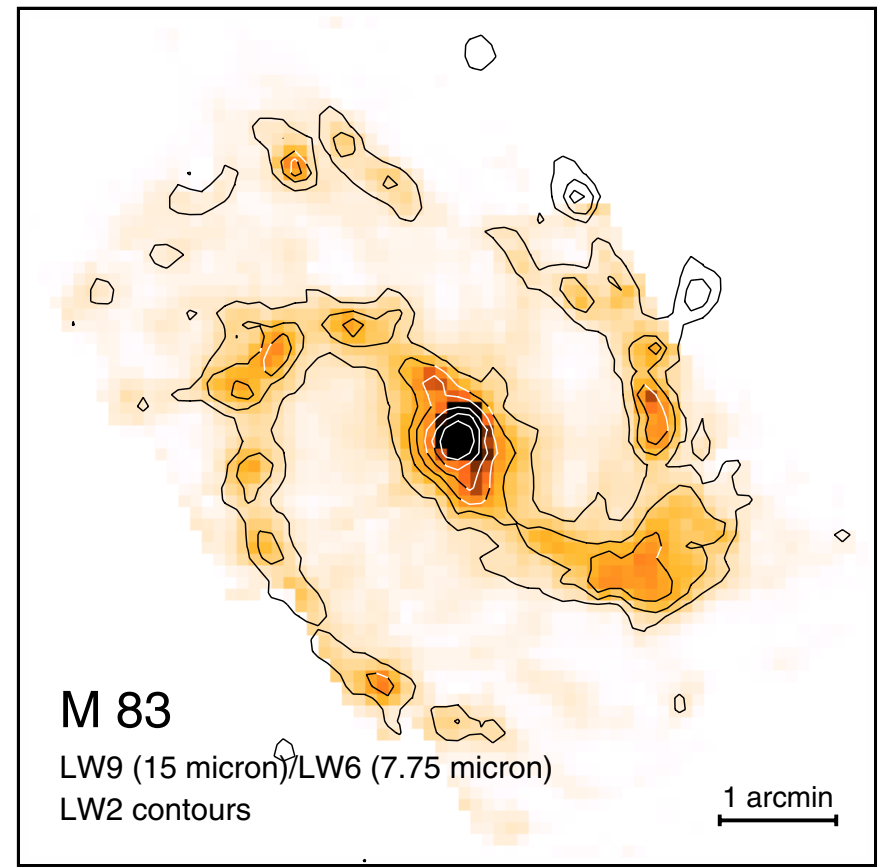

Fig. 5. Map of the ratio of $L W 9 / L W 6(\lambda 15.0 \mu \mathrm{m}$ continuum/ $\lambda 7.75 \mu \mathrm{m}$ PAH).

(e.g. Jourdain de Muizon et al. 1990; Schutte et al. 1993; Allamandola et al. 1999). We normalize each spectrum to the prominent $\lambda 7.7 \mu \mathrm{m}$ PAH band (Fig. 3) and inspect the PAH band ratios in the different regions in M 83. The ratios of the PAH bands $6.2 \mu \mathrm{m}: 7.7 \mu \mathrm{m}: 8.6 \mu \mathrm{m}: 11.3 \mu \mathrm{m}$ vary, at most, by a factor of 1.5 for regions $1,2,3$ and 4 (Table 3 ), which sample very different ISM conditions from the starburst core, to a spiral arm and bulge. The PAH ratios, however, deviate more significantly in the diffuse, interarm region (region 5), with a particularly large value of 0.59 for the $11.3 / 7.7$ ratio, $\sim 2.5$ times larger than toward the nuclear region. Large variations of the 11.3/7.7 ratio have been seen in a wide variety of Galactic and extragalactic sources (Vermeij et al. 2002; Galliano 2004) with no obvious systematic variation with radiation field intensity or hardness (Chan et al. 2001). PAH band ratios vary over only $40 \%$ in reflection nebulae with 3 orders of magnitude variation in radiation field intensity (Uchida et al. 2000). A precise conclusion as to what is controlling these observed variations is not yet clear. Differences in chemical structure, such as compact versus open structures, may be one explanation for the observed variations (Vermeij et al. 2002). Laboratory experiments may eventually shed light on the nature of the observed $\mathrm{PAH}$ band ratios.

\subsection{LW2 and LW3 broad-band images of $M 83$}

Figure 2 shows highly structured MIR emission from the entire optical extent of M 83. The brightest MIR region is coincident with the nucleus of M 83. The emission from the bulge region is extended, at the ISOCAM resolution, along the leading edge of the bar of the galaxy. The south-western and north-eastern ends of the bar are associated with prominent MIR emission regions, which are also present in the radio and in $\mathrm{H} \alpha$ maps (Sect. 4). The MIR morphology argues for the existence of gas and dust in these bar/interstellar medium interfaces.

The inner spiral arm pattern of the galaxy is prominent in the $L W 2$ and $L W 3$ bands (Figs. 1a and 1b) while MIR diffuse emission of lower intensity is detected from the interarm regions. Both $L W 2$ and $L W 3$ images show a lane of enhanced emission in the interarm region between the bulge and the south-eastern inner spiral arm. The "interarm lane" is also visible on the radio image (Fig. 1c) as well as on the optical image (Fig. 2). It is a narrow spiral arm (highly polarized in radio continuum, see Fig. 3 in Beck 2002) with a relatively low starformation rate.

The morphology of the galaxy is very similar in $L W 2$ and $L W 3$, hence, we have chosen the $L W 2$ map for overlays on images of other wavelengths, since the signal-to-noise ratio of the $L W 2$ map is superior to that of the $L W 3$ map.

The ratio of $L W 2 / L W 3$ is known to vary little across the disk of a spiral galaxy and observed to be of the order of 1 in most galaxies (Helou et al. 1996; Vigroux et al 1999; Roussel et al. 2001a; Roussel et al. 2001b). These MIR bands have often been used as a diagnostic along with FIR colors in an ISO-IRAS color-color diagram (Vigroux et al. 1999; Dale et al. 2000). $L W 2 / L W 3 \sim 1$ when the $L W 2$ band is composed completely of PAH bands and the $L W 3$ band has both continuum and PAH band emission, in mostly equal parts. This occurs when the ISM is dominated by neutral regions and photodissociation regions (PDRs), as is the case for much of the disk material in galaxies. Toward H II regions, the rapidly-rising hot dust continuum emission accounts for most of the $L W 3$ band, thus, the $L W 2 / L W 3$ ratio decreases to $\leq 0.5$. This ratio does not vary significantly from normal, quiescent galaxies to mildlyactive galaxies as the IRAS $f(60 \mu \mathrm{m}) / f(100 \mu \mathrm{m})$ increases. However, starburst galaxies and AGNs show a dramatic decrease in the $L W 2 / L W 3$ ratio due to two effects operating simultaneously, the suppression of the PAH band emission in $L W 2$, presumably through the destruction of its carriers, and the dominance of hot $15 \mu \mathrm{m}$ dust continuum emission in $L W 3$ (e.g. Dale et al. 2000; Roussel et al. 2001b). Toward the starburst core and surrounding nuclear region of M 83 (regions 1 and 2) the $L W 2 / L W 3$ ratios are between 0.57 and 0.69 (Table 3), where the continuum emission accounts for 75 to $80 \%$ of the total MIR emission. The ratio for the bulge (region 3) is about $90 \%$, while in regions 4 and 5 the PAH bands contribute $50 \%$ or more to the total MIR emission.

M 83 is a good illustration of the diagnostic capability of the MIR colors. Using the CVF spectra to quantify the components of the $L W 2$ and $L W 3$ broad-bands, we see that the $L W 2 / L W 3$ ratio does provide an accurate tool to probe the $\mathrm{PAH}$ band versus hot dust contributions in the more active regions, such as the nucleus (see also Roussel et al. 2001a,b). However, this broad-band ratio becomes more ambiguous outside of the nuclear region, as the PAH band to continuum ratio increases. In these regions, it is necessary to have other accurate indicators of the MIR components contained within these broad-bands. 
Table 4. Measured fluxes $f_{v}$ and luminosities $L(D=4.5 \mathrm{Mpc})$ in the different MIR filters.

\begin{tabular}{cccccccc}
\hline \hline & $\begin{array}{c}\text { Central } \lambda \\
\mu \mathrm{m}\end{array}$ & $\begin{array}{c}f_{v}\left(d \leq 1^{\prime}\right) \\
\mathrm{Jy}\end{array}$ & $\begin{array}{c}L\left(d \leq 1^{\prime}\right) \\
10^{42} \mathrm{erg} \mathrm{s}^{-1}\end{array}$ & $\begin{array}{c}f_{v}\left(1^{\prime} \leq d \leq 3^{\prime}\right) \\
\mathrm{Jy}\end{array}$ & $\begin{array}{c}L\left(1^{\prime} \leq d \leq 3^{\prime}\right) \\
10^{42} \mathrm{erg} \mathrm{s}^{-1}\end{array}$ & $\begin{array}{c}f_{v}\left(d \leq d_{25}\right) \\
\mathrm{Jy}\end{array}$ & $\begin{array}{c}L\left(d \leq d_{25}\right) \\
10^{42} \mathrm{erg} \mathrm{s}^{-1}\end{array}$ \\
\hline$L W 1$ & 4.5 & 0.6 & 0.4 & 0.8 & 0.6 & & \\
$L W 2$ & 6.7 & 3.0 & 2.4 & 2.5 & 2.0 & 20 & 16 \\
$L W 3$ & 14.3 & 3.8 & 1.2 & 2.9 & 0.9 & 21 & 6.6 \\
$L W 4$ & 6.0 & 1.8 & 0.8 & 1.8 & 0.8 & & \\
$L W 5$ & 6.8 & 2.0 & 0.4 & 2.4 & 0.4 & & \\
$L W 6$ & 7.7 & 4.1 & 1.4 & 4.4 & 1.5 & & \\
$L W 7$ & 9.6 & 2.4 & 0.8 & 2.1 & 0.7 & & \\
$L W 8$ & 11.3 & 4.6 & 0.6 & 4.2 & 0.6 & & \\
$L W 9$ & 14.9 & 3.8 & 0.4 & 2.2 & 0.2 & & \\
\hline
\end{tabular}

\subsection{The ISOCAM strip maps: $\lambda 4.5,7.7$ and $15 \mu \mathrm{m}$}

In general, the strip images reveal similar morphological features as seen in the $L W 2$ and $L W 3$ images. The measured fluxes and luminosities for the individual ISOCAM filters are given in Table 4.

In the following, we will focus on the $L W 1(\lambda 4.0-5.0 \mu \mathrm{m})$, the $L W 6(\lambda 7.0-8.55 \mu \mathrm{m})$ and the $L W 9(\lambda 14.0-16.0 \mu \mathrm{m})$ strip maps.

We show a $L W 9 / L W 6$ ratio map in Fig. 5. The ratio map, overlaid with the $L W 2$ (central wavelength: $\lambda 6.75 \mu \mathrm{m}$ ) contours, shows enhanced $L W 9 / L W 6$ ratios toward regions of high star-formation activity, for example, in the nuclear region and spiral arms. Regions of peaked $L W 2$ emission, which traces the cluster of PAH bands show, in general, enhanced $L W 9 / L W 6$ ratios. The enhanced ratios might be interpreted in terms of (a) increasing densities of the very small grains (VSGs) traced by the continuum of $L W 9$ and (b) possibly increasing temperatures of these grains, if they are in thermal equilibrium. In reality, the VSGs represent grains with a size distribution, and temperature distribution (Désert et al. 1990). On Galactic scales, the hot dust grain emission peaks towards the H II regions, where the PAHs (traced by the LW6 band) show a minimum (e.g. Cesarsky et al. 1996; Verstraete et al. 1996; Henning et al. 1998). PAHs are seen to peak in the photodissociation regions around molecular clouds in the vicinity of star-formation regions.

We also show the $L W 1$ strip map which traces the evolved stellar contribution at $\lambda 4 \mu \mathrm{m}-5 \mu \mathrm{m}$ (Fig. 6). While the bar is also obvious in the $\lambda 4.5 \mu \mathrm{m}$ image, the contrast is not as sharp as in the $L W 2$ or $L W 3$ image, demonstrating the evolved stellar component of the bulge being more dominant over the narrow star-forming bar component (compare Fig. 6 with Figs. 1a and $1 \mathrm{~b}$ ). The differences between the $L W 2$ and $L W 1$ images speak against a strong contribution of stars $(<20 \%$ for such a late type galaxy such as Sc) to the $L W 2$ image, as has been argued before (e.g. Boselli et al. 2003).

\section{The radio view of $\mathrm{M} 83$}

The $\lambda 6 \mathrm{~cm}$ radio continuum map (total intensity) derived from VLA and Effelsberg data is presented in Fig. 1c. The radio

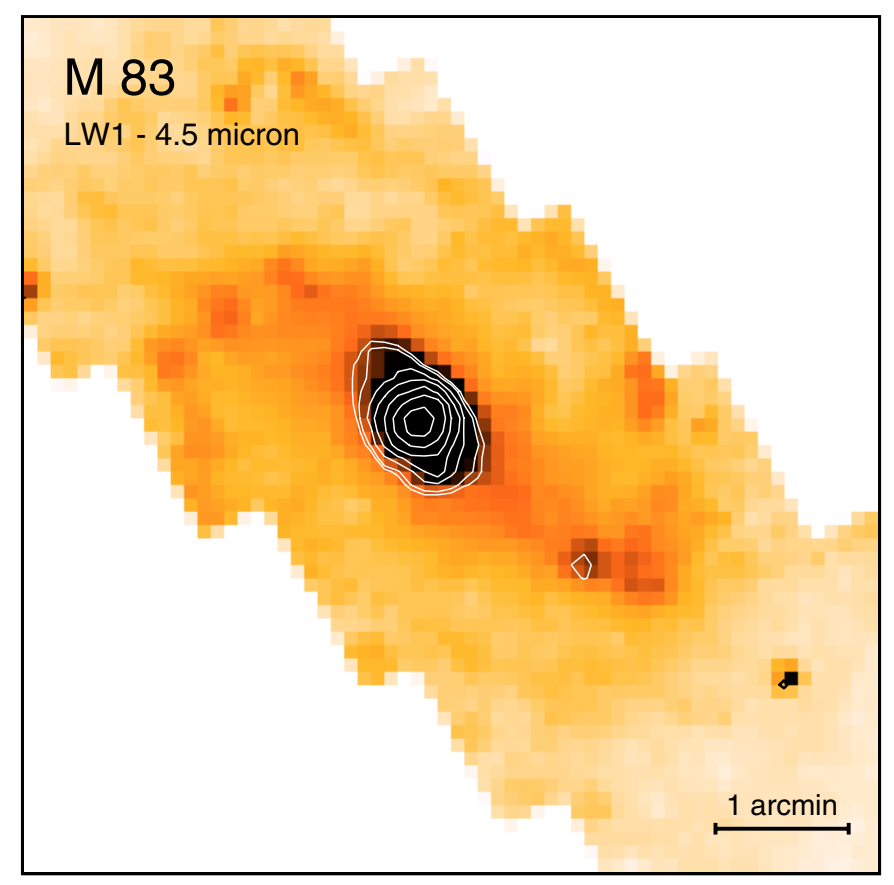

Fig. 6. ISOCAM $L W 1(\lambda 4.5 \mu \mathrm{m})$ image; regions of higher intensity have been sketched with contours.

emission is dominated by the non-thermal synchrotron process. Neininger et al. (1993) found that the ratio between large-scale non-thermal and thermal emission at $\lambda 2.8 \mathrm{~cm}$ varies from $\simeq 3$ in the inner region of $M 83$ to $\simeq 10$ in the outer parts. However, the thermal fraction may increase locally in star-forming regions of spiral arms. Our new separation of thermal and non-thermal emission at $23^{\prime \prime}$ resolution (see Sect. 7.1) reveals a thermal fraction at $\lambda 6 \mathrm{~cm}$ which is $25 \%$ on average, but up to $70 \%$ locally in spiral arms. Very similar results were obtained for the spiral galaxy NGC 6946 (Walsh et al. 2002).

The synchrotron intensity, $I_{\text {sync }}$, is proportional to $N_{\mathrm{CRE}} B^{1+\alpha_{\mathrm{n}}}$ where $N_{\mathrm{CRE}}$ is the energy density of cosmic-ray electrons in the relevant energy range, $B$ is the strength of the total field component in the sky plane, and $\alpha_{\mathrm{n}}$ is the non-thermal spectral index (defined as $I_{\text {sync }} \propto v^{-\alpha_{\mathrm{n}}}$ ). In case of (often assumed) energy density equipartition between cosmic rays and magnetic fields, $I_{\text {sync }} \propto B^{3+\alpha_{n}}$. Thus synchrotron 


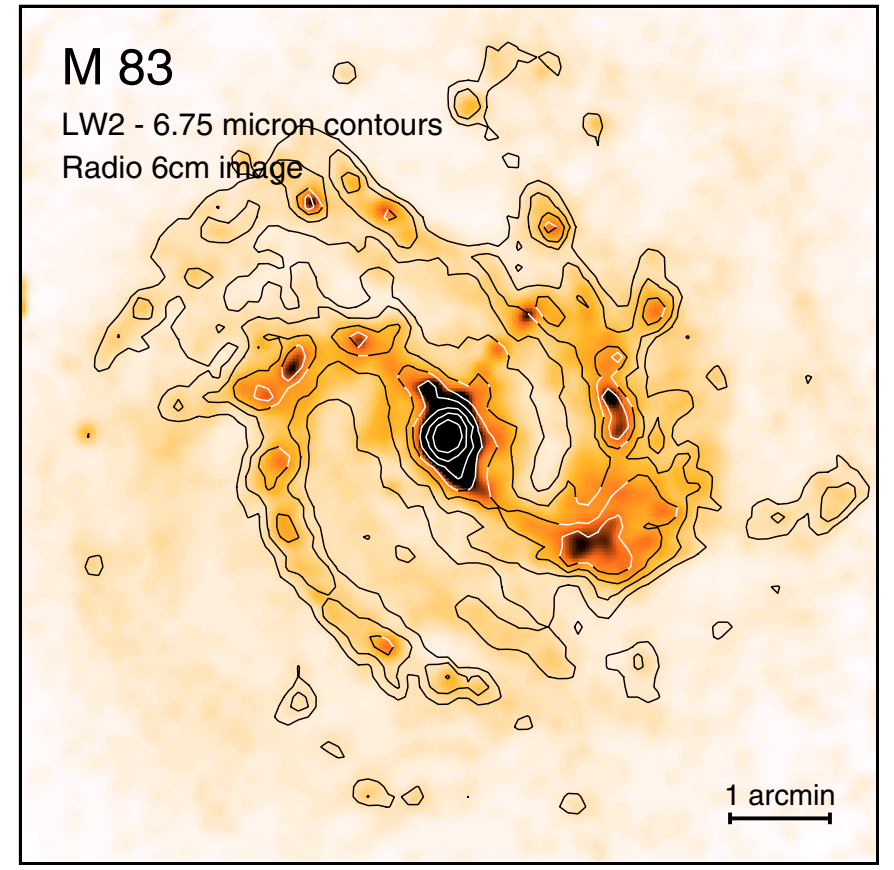

Fig. 7. Contours of the ISOCAM $L W 2(\lambda 6.75 \mu \mathrm{m}$ central wavelength; angular resolution $6^{\prime \prime}$ ) image overlaid onto the $\lambda 6 \mathrm{~cm}$ radio continuum image (resolution 10").

intensity is a sensitive measure of field strength and its variations.

Emission from the bar is concentrated toward the outer edge (i.e. leading with respect to the galaxy's rotation). This can be regarded as a signature of a shear shock due to gas infall on the inner side of the bar (see numerical models e.g. by Athanassoula 1992). If the magnetic field is compressed together with the gas, a compression ratio of 4 in a strong adiabatic shock should result in an increase in synchrotron intensity of a factor of 16 if the density of cosmic-ray electrons is constant (assuming $\alpha_{\mathrm{n}} \simeq 1$, see Sect. 7.1). This factor could be larger if the cosmic-ray density also increases in the shock (energy equipartition). However, the intensity contrast between the radio bar and its surroundings is only $\simeq 3$, similar to NGC 1097 , a galaxy with a larger bar (Beck et al. 1999). Either the shock generates magnetic turbulence, or the magnetic field is not frozen into the gas flow, or the shock has a complex 3-D structure. Better numerical models, which take magnetic fields into account, are required.

Figure 7 demonstrates the close association between the radio emission and the MIR dust emission. This is most obvious in the spiral arm 2 to $3^{\prime}$ north of the center, where both the radio continuum and dust distribution reveal a sharp edge while the optical spiral arm extends much further to the north.

Three sources, which are not obvious in the MIR images but appear in the radio map at 0.4-1.'4 north-west of the center (Fig. 7), are supernova remnants and/or background quasars. Their positions do not coincide with any of the observed 20th century supernova events. In principle, supernova remnants and dusty quasars have been detected at MIR wavelengths with ISOCAM (e.g. Lagage et al. 1996; Arendt et al. 1999; Leech et al. 2001). While it is conceivable that a hint of

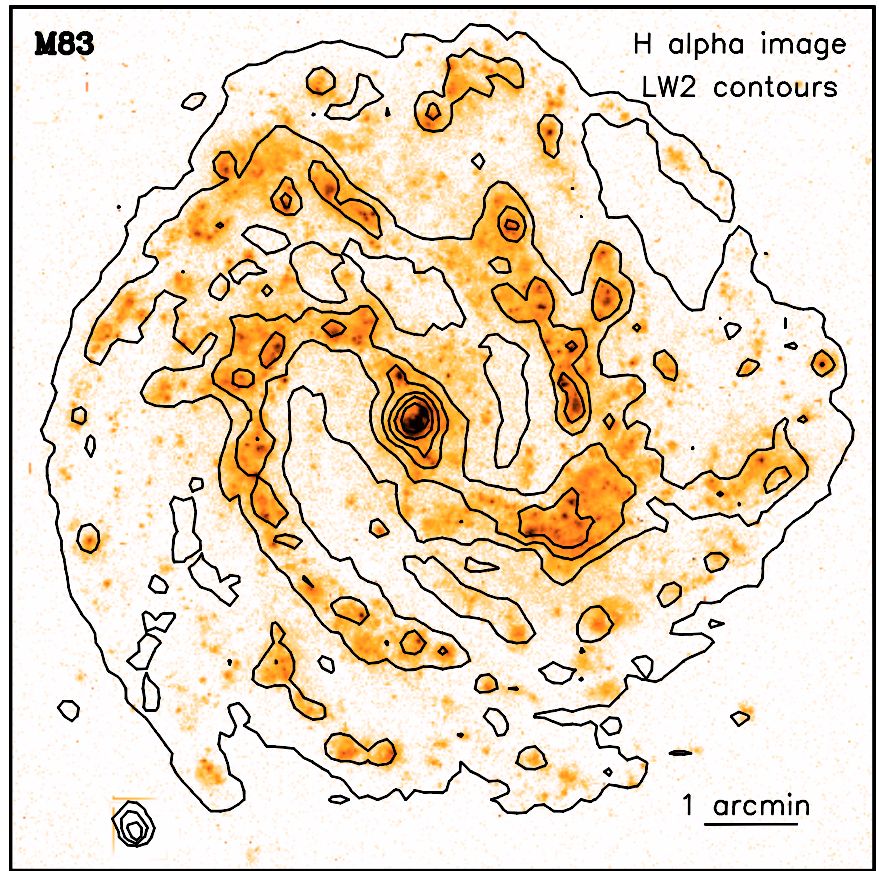

Fig. 8. $L W 2\left(\lambda 6.75 \mu \mathrm{m}\right.$ central wavelength; angular resolution $\left.6^{\prime \prime}\right)$ contours overlaid onto the $\mathrm{H} \alpha$ image of Lundgren et al. (2005).

MIR emission is detected toward these radio sources, they are not detectable at the sensitivity and angular resolution of these images.

The close correlation between radio continuum and midinfrared dust emission is indeed striking in M 83 (Figs. 7 and 1a-c) and in NGC 6946 (Frick et al. 2001; Walsh et al. 2002). The explanation is far from being trivial (see Sect. 7.3).

\section{Comparison of the MIR morphology with further tracers of the interstellar medium}

\subsection{H $\alpha$ emission}

The $\mathrm{H} \alpha$ image of M 83 was initially presented in Lundgren et al. (2005). The FWHM of the PSF is $1{ }^{\prime \prime} 18$ (30 pc). The calibrated $\mathrm{H} \alpha$ image (Fig. 1d) is used here for quantitative comparison with the radio and MIR emission, both of which have spatial resolutions poorer than that of $\mathrm{H} \alpha$.

Figure 8 presents the $L W 2$ contours overlaid on the $\mathrm{H} \alpha$ image of Lundgen et al. (2005). In general, the MIR emission and $\mathrm{H} \alpha$ emission highlight the same region, but differences exist, e.g. in the north-east (Sect. 4). The spiral arms are traced well at both wavelengths, and many of the bright $\mathrm{H} \alpha$ emission regions correspond to local maxima of the MIR emission. Diffuse $\mathrm{H} \alpha$ emission is also present throughout the interarm region. The correlation between MIR and $\mathrm{H} \alpha$ is discussed in Sect. 7.4.

\subsection{Diffuse $X$-ray emission}

M 83 was observed with the Einstein (Trinchieri et al. 1985), GINGA (Ohashi et al. 1990), ASCA (Okada et al. 1997) ROSAT (Ehle et al. 1998; see Fig. 9; Immler et al. 1999) and Chandra (Soria \& Wu 2002; Soria \& Wu 2003) 


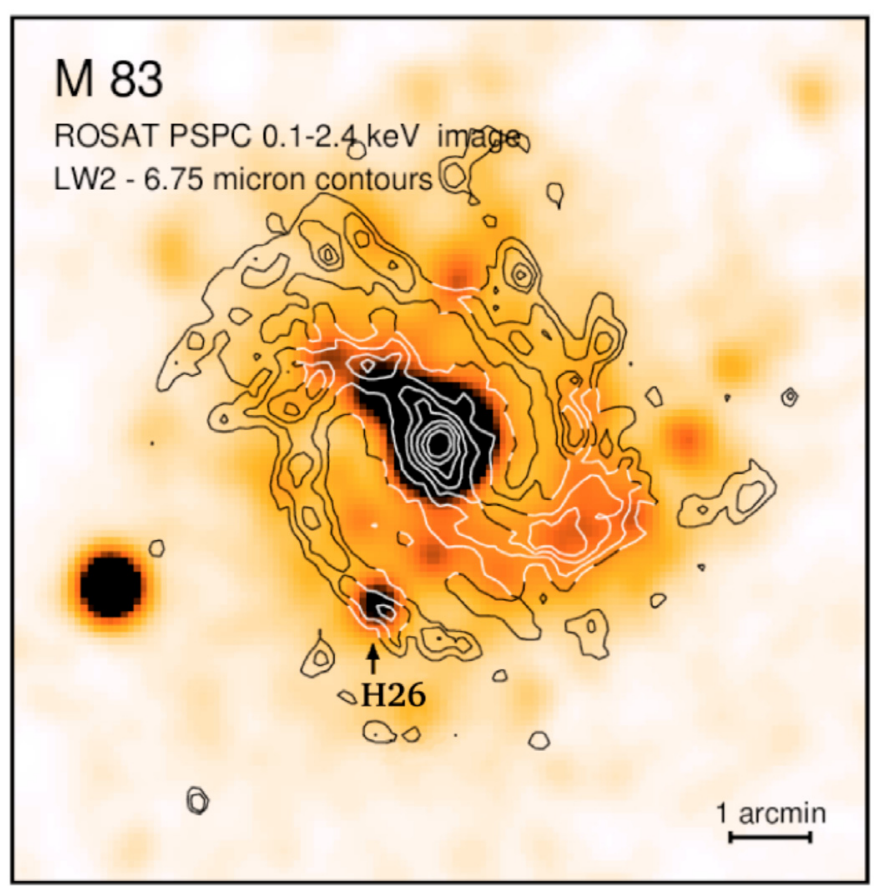

Fig. 9. $L W 2\left(\lambda 6.75 \mu \mathrm{m}\right.$ central wavelength; angular resolution $\left.6^{\prime \prime}\right)$ contours overlaid onto the ROSAT PSPC 0.1-2.4 keV image from Ehle et al. (1998) with an energy-dependent angular resolution of $25^{\prime \prime}-52^{\prime \prime}$. $\mathrm{H} 26$, labeled in the figure, is an X-ray luminous source coinciding with a MIR peak in a spiral arm. It is identified as a supernova remnant candidate (Ehle et al. 1998).

observatories. Diffuse X-ray emission was detected by ROSAT as well as by Chandra. From spectral investigations, roughly $65 \%$ of the diffuse X-ray emission was attributed to a million degree component of the ISM, while the remaining part was attributed to unresolved point sources. While Chandra was well suited to clearly resolve the starburst nucleus region (see Sect. 6.2) for the first time and to detect the discrete source population in M 83, the diffuse extended emission could be traced (after spatial smoothing) only from the optically bright spiral arms.

ROSAT PSPC, in contrast, showed extended X-ray radiation from almost the whole optically visible part of that galaxy. Ehle et al. (1998) interpreted the diffuse X-ray emission as a multi-temperature thermal plasma: a soft X-ray emitting spherical halo component and a hotter disk component, dominating the emission in the harder $0.5-2.0 \mathrm{keV}$ ROSAT band.

\subsection{Molecular gas}

Comparison of the distribution of the $\operatorname{CO}(J=1-0)$ emission at $22^{\prime \prime}$ angular resolution (Lundgren et al. 2004a) with that of the MIR dust emission shows very good spatial agreement in the disk (see Fig. 10). However, there are also displacements: local peaks (or in some cases crests) of $\operatorname{CO}(J=1-0)$ emission appear to be shifted about $10-20^{\prime \prime}$, but there is no apparent systematic trend such that the $\operatorname{CO}(J=1-0)$ emission lies outside the MIR emission or vice verse. The local maxima of the MIR emission are better aligned with those of the $\mathrm{CO}(J=2-1)$ emission with 14" resolution (Lundgren et al. 2004a). This is

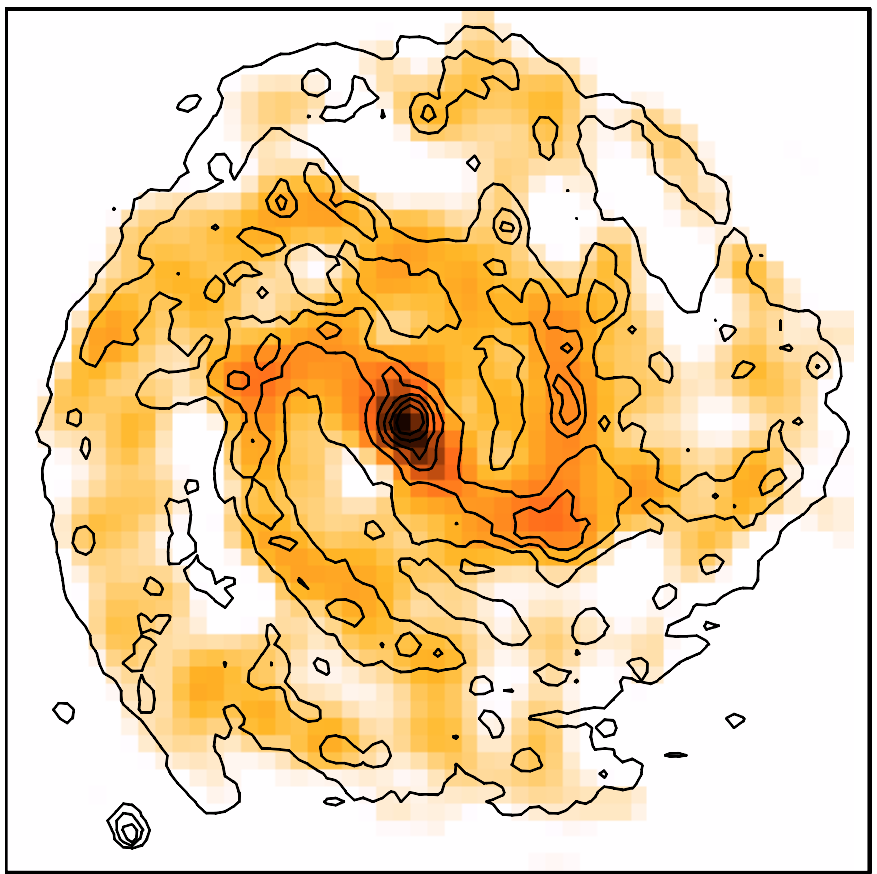

Fig. 10. $L W 2\left(\lambda 6.75 \mu \mathrm{m}\right.$ central wavelength; angular resolution $\left.6^{\prime \prime}\right)$ contours onto the $\mathrm{CO}(J=1-0)$ image of Lundgren et al. (2004a) with a resolution of $22^{\prime \prime}$.

perhaps not so surprising since molecular gas clouds in regions with active star formation are likely to have an increased kinetic temperature (and perhaps also higher density), resulting in stronger emission from higher- $J$ emission lines.

The central peak in the MIR data agrees well with the IR center position (Gallais et al. 1991). The nucleus appears as a single peak in the MIR data and does not have the doublepeaked nature seen in the $\mathrm{CO}(J=1-0)$ (Handa et al. 1994), $\mathrm{CO}(J=2-1)$ (Lundgren et al. 2004a) and $\operatorname{CO}(J=3-2$ and $J=4-3$ ) data (Petitpas \& Wilson 1998). See Sect. 6.2 for further discussion of the nuclear region.

Additionally, the nuclear and bar region, as well as the south-eastern spiral arm, were observed in CO line emission with high resolution (Handa et al. 1990; Lord \& Kenney 1991; Rand et al. 1999; Dumke et al. 2005). These observations will be discussed in Sects. 6.2 and 6.3, respectively.

\section{Discussion}

\subsection{Radial brightness profiles of different tracers of the interstellar medium}

The radial extent of different tracers of the neutral, molecular and ionized ISM was compared. Figure 11 shows the profiles for seven tracers: $R$ band (Larsen \& Richtler 1999), MIR (LW2 and $L W 3$ ), $\mathrm{H} \alpha$ (Lundgren et al. 2005), $\mathrm{CO}(J=1-0)$ (Lundgren et al. 2004a), radio continuum $(\lambda 6 \mathrm{~cm})$, and H I (Tilanus \& Allen 1993). The profiles have been calculated from 23 " resolution maps, which were corrected for distortion due to the inclination of the galaxy $\left(24^{\circ}\right)$, by adjusting the distance scale along the minor axis by $1 / \cos (24)$.

For all profiles, except that of $\mathrm{HI}$, the intensity declines from the central region out to $r \simeq 1^{\prime}$, where most profiles have a 


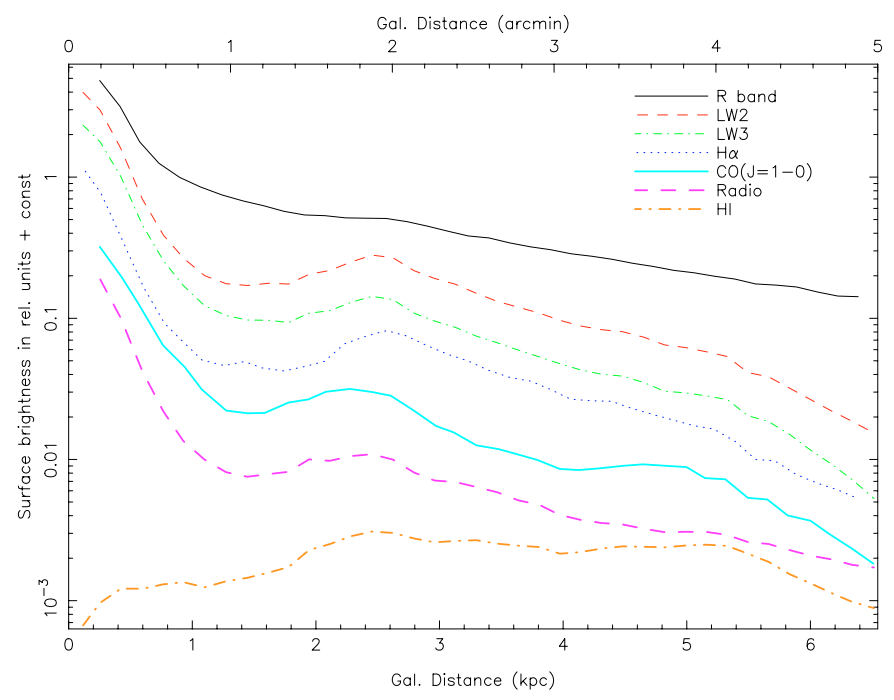

Fig. 11. Radial intensity profiles versus galactocentric radius for different tracers in M 83.

local minimum and a corresponding local maximum at $r \simeq 2^{\prime}$. In the radial range $2^{\prime}-4^{\prime}$ all tracers, except $\mathrm{HI}$, show an exponential decline. The MIR and $\mathrm{H} \alpha$ profiles seem to be similar, except that the MIR profiles near $2^{\prime}$ have more pronounced peaks, and beyond $4^{\prime}(5 \mathrm{kpc})$, the MIR profile shows a steeper decline. We find a scale length of $1: 3 \pm 0,1$ ( $=1.7 \pm 0.1 \mathrm{kpc}$ ) for the $\mathrm{H} \alpha$ and the MIR. The same value was found for the thermal radio continuum emission at $\lambda 2.8 \mathrm{~cm}$ (Ehle et al. 1998). These authors also found the same scale length $\left(1^{\prime} 3 \pm 0.1\right)$ for the hard $\mathrm{X}$-ray emission from the disk for $r>2 ! 1(r>3 \mathrm{kpc})$.

In the optical wavelength region (here represented by the $R$ band), $\mathrm{CO}(J=1-0)$ and total radio continuum, the scale lengths are considerably larger: $2 \cdot 2 \pm 0,1,2{ }^{\prime} 1 \pm 0{ }^{\prime} 3$ and $1{ }^{\prime} \cdot 7 \pm 0{ }^{\prime} 1$, respectively. The fact that $\mathrm{H} \alpha$ has a smaller scale length than that of the stellar light has previously been noted in M 83 by Larsen \& Richtler (1999). According to Ryder \& Dopita (1994), however, the $\mathrm{H} \alpha$ scale length in galaxies is usually larger that that of the stellar broad-band emission. The explanation for M 83 probably lies in the fact that the central region and the inner spiral arms are undergoing bursts of star formation.

For $r>4^{\prime}(r>5 \mathrm{kpc})$ the decline of the MIR and $\mathrm{H} \alpha$ emission steepens while that of the $R$ band and radio continuum continues without a break. The scale lengths in the radial region $4^{\prime} .0-5^{\prime} \cdot 0$ are $0.7 \pm 00^{\prime} 1,00^{\prime} 6 \pm 0 .^{\prime} 1$ and $0^{\prime} \cdot 9 \pm 00^{\prime} 1$ for $L W 2, L W 3$ and $\mathrm{H} \alpha$, respectively, while the $R$ band and radio continuum have scale lengths of $2{ }^{\prime} 6 \pm 0.2$ and $1: 7 \pm 0,1$, similar to those in the inner disk. The scale length of the $\operatorname{CO}(J=1-0)$ cannot be determined reliably since this radial range lies partially outside the area which was covered in Lundgren et al. (2004a). The large scale length in radio continuum is known to exist in many spiral galaxies, e.g. in NGC 6946 (Walsh et al. 2002), and is an effect of the diffusion of cosmic-ray electrons away from the star-forming regions and the large extent of magnetic fields.

For $\mathrm{HI}$, the decline begins only beyond $5.4 \mathrm{kpc}$ and is in good agreement with the existence of a giant $\left(r>40^{\prime}\right) \mathrm{H} \mathrm{I}$ halo for M 83 (Huchtmeier \& Bohnenstengel 1981).
In the nuclear region all of the tracers except HI reach brightness maxima which are most pronounced in $\mathrm{H} \alpha$ and the radio. Active star formation can convert $\mathrm{H} \mathrm{I}$ into molecular and ionized gas. From a comparison of the MIR and radio data, we conclude that we have a higher radio/MIR intensity ratio for the nucleus than for the disk. While a part of this effect may be due to ISOCAM saturation effects, we may also argue for a compression of the magnetic field by the bar shock, enhancing the radio (synchrotron) emission. Alternatively, the coupling between magnetic fields and gas clouds may be more effective in the nuclear region, e.g. due to higher gas temperatures. Such a higher temperature is indicated by increasing $\mathrm{CO}(J=4-3) / \mathrm{CO}(J=3-2)$ line ratios in the nuclear region (Petitpas \& Wilson 1998).

The steeper decline of the MIR intensity versus the optical or H I brightness in our data is in good agreement with results of Roussel et al. (2001a) who find the MIR disks in spiral galaxies are smaller than the optical or H I disks. We further confirmed this trend with the help of the MIR strip observations which cover 17 ' $^{\prime} \times 5^{\prime} \cdot 4$, versus the $12^{\prime} .8 \times 12^{\prime} .8$ field of the $L W 2 / L W 3$ observations. Therefore, our findings cannot be explained in terms of difficulties of the background estimation in case of the $L W 2$ and $L W 3$ maps which almost completely cover the optical extent of M $83\left(d_{25}=11{ }^{\prime} 5\right)$.

\subsection{The nuclear and bar region}

To further understand the nature of the nuclear MIR and radio emission, we zoom into the central $2^{\prime} \times 2^{\prime}$ region $(2.6 \mathrm{kpc} \times$ $2.6 \mathrm{kpc})$ of M 83 and investigate their relationship with various ISM tracers, such as $\mathrm{H} \alpha, \mathrm{HI}, \mathrm{CO}(J=1-0), \mathrm{CO}(J=2-1)$ and $\mathrm{CO}(J=3-2)$ (Fig. 12). We have also sketched in Fig. 12f the NIR emission features (Adamson et al. 1987; Gallais et al. 1991; Sofue \& Wakamatsu 1994; Elmegreen et al. 1998; Thatte et al. 2000), namely the starburst shells, the double nucleus and the mini-bar.

From the $\mathrm{H} \alpha$ morphology (Fig. 12a), it is clear that the bulge region is not a point source, but shows two "bubble-like" structures opening out to the north-west and south-east of the nucleus. While the MIR image cannot resolve the nuclear emission region due to its lower angular resolution, there is good coincidence of MIR and $\mathrm{H} \alpha$ emission on a larger scale toward the central region. Both tracers peak at the nucleus of the galaxy. Bright $\mathrm{H} \alpha$ maxima at the position angle of $10^{\circ}$ and $190^{\circ}$ at distances from the nucleus of $\sim 30^{\prime \prime}(650 \mathrm{pc})$ and $\sim 20^{\prime \prime}-30^{\prime \prime}$, respectively, show that the optical bar alignment and star formation activity along and at the ends of the bar are traced by "tongues" of the $L W 2$ contours. The $L W 2$ contours also outline the compact $\mathrm{H} \alpha$ regions at the position angle of $270^{\circ}$ and $335^{\circ}$ (distances of $\sim 40^{\prime \prime}$ and $\sim 35^{\prime \prime}$, respectively).

Figure $12 \mathrm{~b}$ displays the $\lambda 6 \mathrm{~cm}$ radio continuum image and red contours and the LW2 contours in green. The MIR observations and the radio observations do not provide sufficient angular resolution to detect a possible correlation with the NIR emission components (sketched in Fig. 12f). The nucleus itself is not resolved in either image, but "tongue-like" 

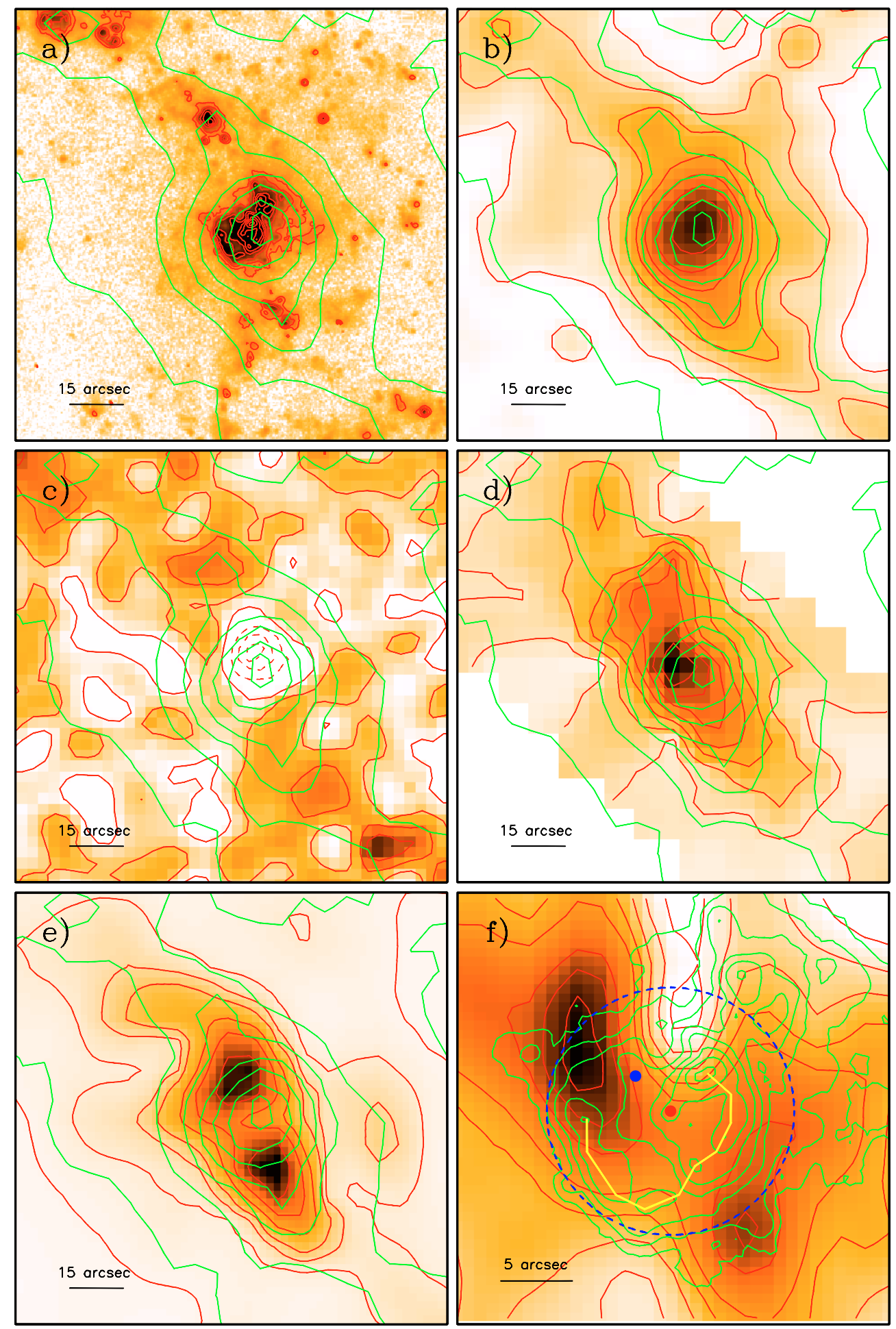

Fig. 12. The nuclear region of of $M 83$. The scales of a) to e) are identical, while f) is zoomed in by a factor of about 3 with respect to the other images. In a) to e) the green contours show the ISOCAM $L W 2$ data $\left(\lambda 6.75 \mu\right.$ m central wavelength, resolution $\left.6^{\prime \prime}\right)$, while the background image and the red contours represent the following: a) $\mathrm{H} \alpha$ emission (logarithmic color scale, angular resolution 1". 1, Lundgren et al. 2005) b) $\lambda 6 \mathrm{~cm}$ radio continuum emission (logarithmic color scale, resolution 10") c) H I column density (Tilanus \& Allen 1993, linear color scale, resolution $\left.12^{\prime \prime}\right)$. Note that the HI line is seen in absorption towards the center of the galaxy. This is represented with dashed contours. d) $\mathrm{CO}(J=1-0)$ emission (Handa et al. 1990, linear color scale, resolution 16"). e) $\mathrm{CO}(J=2-1)$ map (Lundgren et al. 2004a, linear color scale, resolution 14"). In f) the green contours represent $\mathrm{H} \alpha$ emission, and they are superposed onto an CO(3-2) map (Petitpas \& Wilson 1998, linear color scale, resolution, resolution $\left.14^{\prime \prime}\right)$. The yellow line sketches out the star forming arc. The blue and the red dots are the bright peak seen in the $K$ band, and the center of symmetry of the $K$ band isophotes, respectively (Thatte et al. 2000). The blue dashed circle represents the ring seen in the $J-K$ images (Elmegreen et al. 1998). 
structures of the contours from the nucleus following the bar to the south and north are visible in both bands.

A correspondence of the HI void in the nuclear area (dashed red contours) and the MIR emission peak is shown in Fig. 12c. The tongue-like $L W 2$ emission features (green contours) south-west and north-east of the nucleus coincide with local H I maxima.

Our Fig. 12d shows the $\mathrm{CO}(J=1-0)$ image and red contours (Handa et al. 1990) in comparison with $L W 2$ contours (green). Both tracers peak on the nucleus of the galaxy. The $\mathrm{CO}(J=1-0)$ contours do not show the "bottleneck" structure at $\sim 30^{\prime \prime}(650 \mathrm{pc}) \mathrm{NE}$ and $\mathrm{SW}$ of the nucleus. If real, and not an effect due to the large spacing $\left(15^{\prime \prime}\right)$ of the CO map, this might further indicate that we indeed trace different components: the molecular gas along the bar and the MIR PAH emission along the optical spiral arms and leading edge of the bar.

As shown in our overlay of the MIR contours (resolution $\left.6^{\prime \prime}\right)$ on the $\operatorname{CO}\left(J=2-1\right.$, resolution $\left.14^{\prime \prime}\right)$ data (Fig. 12e), we can exclude the presence of a double-peaked MIR structure separated by $\sim 20^{\prime \prime}(430 \mathrm{pc})$ as detected in the $\mathrm{CO}(J=2-1)$ (Lundgren et al. 2004a) and $\mathrm{CO}(J=3-2)$ (Petitpas \& Wilson 1998) data. The MIR peak appears to lie in the middle of the CO peaks. Similar to the $\operatorname{CO}(J=2-1)$, the contours of the $L W 2$ emission are located at the leading edge of the bar, which argues for the presence of dust lanes along the leading edges of the bar. Recent $\mathrm{CO}(J=3-2)$ data from the Heinrich Hertz Telescope (HHT) (Dumke et al. 2005) also show this. These results confirm the conclusion of Elmegreen et al. (1998) who have demonstrated this point using $J-K$ color images.

To obtain further insight on the nature of the nuclear emission beyond the resolution of the MIR and radio continuum data, we overlaid the $\mathrm{H} \alpha$ contours on the $\mathrm{CO}(J=3-2)$ image (Fig. 12f), zooming in further. In this figure we sketched the two near-infrared nuclei (blue and red dots), the star forming arc (yellow) as well as the near infrared ring (blue-dashed ring) seen in the $J-K$ images (Elmegreen et al. 1998). The brightest $\mathrm{H} \alpha$ emission peak is close to the bright near-infrared peak of the galaxy (blue dot). Both $\mathrm{H} \alpha$ emission peaks lie on the ring seen in the $J-K$ images, while the center of symmetry of the $K$-band emission lies at the center of the image (red dot; Thatte et al. 2000), between the two $\mathrm{H} \alpha$ emission peaks. Optical spectroscopy of these metal-rich H II regions is given in Bresolin \& Kennicutt (2002). For the emission peak at the position of the north-eastern nucleus, a Wolf-Rayet bump is detected and the age of the $\mathrm{H}$ II region is approximately $5 \mathrm{Myr}$.

Two bubble-like structures of ionized gas are visible to the NW and SE, both oriented nearly perpendicular to the major axis and bar of M 83. These structures are embedded in large-scale, diffuse $\mathrm{H} \alpha$ emission with an extent of $18^{\prime \prime} \times 12^{\prime \prime}$ $(390 \mathrm{pc} \times 260 \mathrm{pc})$. While star-forming regions of different intensities or a double or multiple nucleus can, in principle, explain the morphology of the $\mathrm{H} \alpha$ emission peaks, one might be tempted to speculate about the detection of an inflow- or outflow/superwind-like $\mathrm{H} \alpha$ morphology in the central region of the galaxy as known to exist for other galaxies with nuclear starburst activities (e.g. Bland \& Tully 1988; Lehnert et al. 1999 for M 82, or Schulz \& Wegner 1992 for NGC 253). Dense gas and dust present in spiral arms, compressed by spiral density waves can also affect the morphology of the $\mathrm{H} \alpha$ emission.

The nuclear region of M 83 could clearly be resolved with the advent of Chandra observations (Soria \& Wu 2002). A highly structured composition of 15 discrete sources and unresolved clump-like features due to faint sources or hot gas clouds was discovered. The nuclear X-ray emission is strongest inside the outer dust ring (Elmegreen et al. 1998) and contains both the IR photometric nucleus (consistent with the second brightest Chandra-detected nuclear X-ray source) and the starforming arc (in the south). While the optical emission is more strongly concentrated around the IR nucleus and along the starforming arc, the X-ray emission is more uniformly distributed and extends to larger galactocentric radii: to the south-west along the main galactic bar and also towards the north-west across a dust lane (part of the outer circumnuclear ring) and in the direction perpendicular to the bar. X-ray emission, uncorrelated with any bright (star-forming) optical regions, was also found to the east of the IR photometric nucleus and might originate from remnants of stellar evolution. The X-ray spectrum of the unresolved nuclear X-ray emission suggests that the ISM has been enriched by the ejecta of type II supernovae and stellar winds (see also Soria \& Wu 2002).

\subsection{The south-eastern spiral arm}

The south-eastern spiral arm of M 83 was studied in detail at several wavelengths. The atomic and ionized hydrogen in this arm region was observed by Tilanus \& Allen (1993). Deutsch $\&$ Allen (1993) focused on the non-thermal radio emission and $\mathrm{H} \beta$ emission. $\mathrm{CO}(J=1-0)$ observations were carried out by Lord \& Kenney (1991) and Rand et al. (1999). Rand et al. (1999) stress, as indicated already by the observations of Lord \& Kenney (1991), that the CO emission of the arm was found to be aligned with a dust lane delineating the inner edge of the spiral arm, but beyond a certain point along the arm the emission shifts downstream from the dust lane. While the CO emission at least partially covers the dust lane, the H I emission along the arm is offset from the dust lane to the east by up to $700 \mathrm{pc}$. Tilanus \& Allen (1993) propose a diffuse shock or dissociation scenario to explain the H I findings. Rand et al. (1999) discuss heating of the $\mathrm{CO}$ gas by UV radiation from young stars, heating by low energy cosmic rays or an interstellar medium of different density components, in order to explain the offset of the $\mathrm{CO}$ emission from the dust lane.

In our Figs. 13a-d, we present the contours of the CO map as shown by Rand et al. (1999) in their Fig. 2 overlaid on the $L W 2, \mathrm{HI}$, radio continuum and VLT/FORS1 $B$ band (ESO archive) images. The $L W 2$, $\mathrm{HI}$ and radio data were rebinned to a pixel size of $1^{\prime \prime}$ for the comparison with the $\mathrm{CO}(J=1-0)$ contours. The $\mathrm{CO}$ emission lies along a sharp dust lane visible in blue images of M 83 in most of the region displayed, but near a certain point the emission falls below the detection limit, and this point is marked by an arrow in Fig. $13 \mathrm{~b}$. It is worth mentioning that the non-detection of $\mathrm{CO}$ emission coincides with a local maximum in the $\mathrm{HI}$ emission, corresponding to a column density of $2.2 \times 10^{21} \mathrm{~cm}^{-2}$ at the 


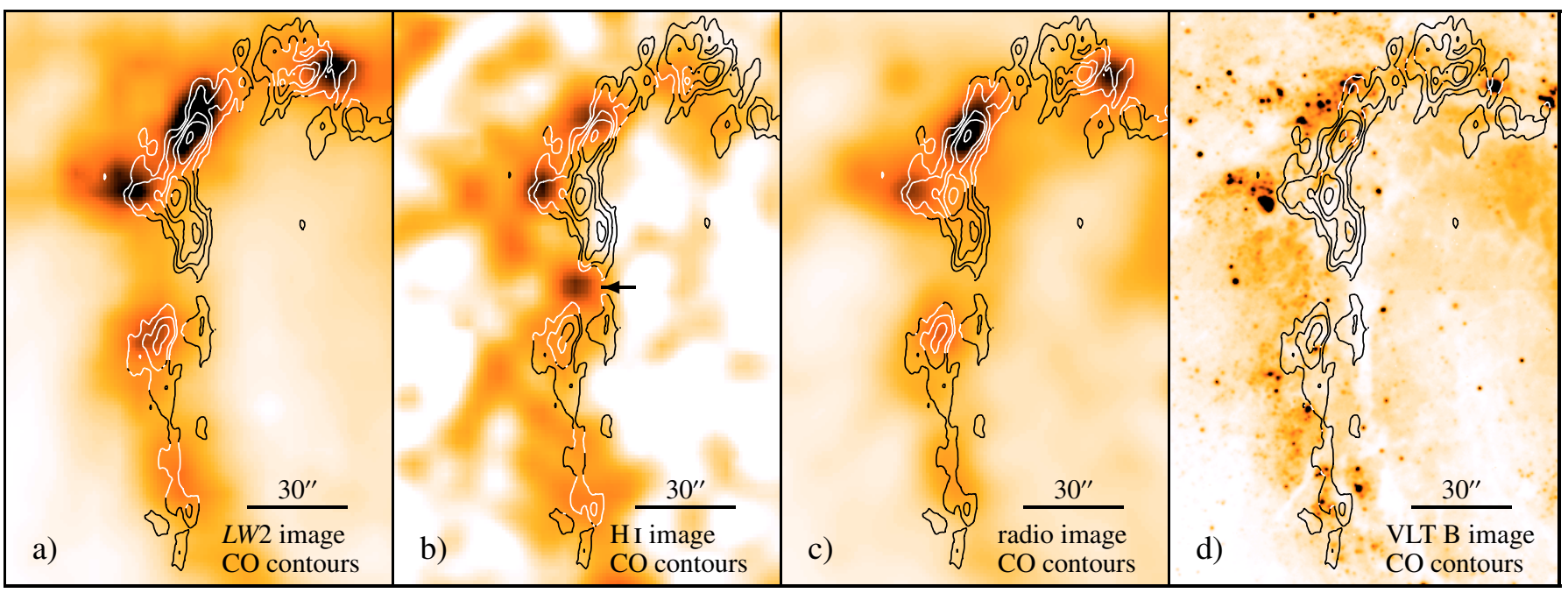

Fig. 13. South-eastern spiral arm of M 83: a) Contours of the CO emission on the ISOCAM LW2 image, b) H I image of Tilanus \& Allen (1993) and contours of $\mathrm{CO}, \mathbf{c})$ the radio continuum map with CO contours and d) the VLT/FORS1 B image (ESO archive) and CO contours. The first three images were rebinned to a pixel size of $1^{\prime \prime}$ for this representation. The CO map is from Rand et al. (1999), and we show the same contour levels as in their publication (their Fig. 2).

resolution of the HI map or an average density above $1.7 \times$ $10^{21} \mathrm{~cm}^{-2}$ for an integration radius of 7 '. 5 . We detect neither an enhancement in MIR nor in radio continuum emission at this location. Further to the south, the $\mathrm{CO}$ emission bifurcates, with a local maximum to the west. The emission is aligned with a dust lane and an elongated emission feature following the arm structure as highlighted by the highest MIR and radio intensities. This alignment might argue for enhanced star formation and cosmic ray densities (Rand et al. 1999) or strong magnetic fields along the $\mathrm{CO}$ filament extending from the local $\mathrm{H}$ I maximum marked in Fig. $13 \mathrm{~b}$ to the south.

However, the OVRO $\operatorname{CO}(J=1-0)$ data is not a reliable source to trace the bulk of molecular gas since it only reveals $2-5 \%$ of the total $\mathrm{CO}(J=1-0)$ emission in this region (see Rand et al. 1999). It is likely that the emission that was detected has its origin in clouds that are heated or compressed by star formation and/or shocks, instead of tracing the cool molecular gas. As an example, the OVRO data traces the leading edge of the molecular bar, and not the bar itself (see Fig. 5 in Lundgren et al. 2004a). Furthermore, we should keep in mind that this may also affect the comparison with the $\mathrm{HI}$ data, where $55 \%$ of the total H I emission within the Holmberg radius was detected (Tilanus \& Allen 1993).

\section{Correlations between different tracers of the interstellar medium}

\subsection{The method}

The correlations are determined using an algorithm that plots corresponding values with linear or logarithmic axes (Nieten 2001), using different gridding and weighting schemes. The results presented here were obtained with a $1 / \sigma$ weighting, where $\sigma$ is the rms noise in each image. Only intensities with amplitudes above $5 \sigma$ were used in order to avoid any influence of instrumental noise. Only spatially independent points are selected. Selecting pixels separated by 2.0 beam widths, at which point the Gaussian telescope beam overlaps by only $2 \%$, is a good compromise between independence and maximal data usage. Four different hexagonal grids were used for each correlation to assess the effect of gridding on the resulting correlations, and their mean value was adopted. The difference in the correlation coefficient for slightly offset hexagonal grids is typically a few percent.

We used the MIR, $\mathrm{H} \mathrm{I}, \mathrm{CO}, \mathrm{H} \alpha$ and $\lambda 6 \mathrm{~cm}$ radio continuum maps for this correlation study. The radio intensity was separated into its thermal and non-thermal components with help of a spectral index map between $\lambda 20 \mathrm{~cm}$ (Sukumar \& Allen 1989) and $\lambda 6 \mathrm{~cm}$, assuming constant spectral indices for the thermal ( $\alpha=0.1)$ and non-thermal ( $\alpha=1.0)$ components, as in Neininger et al. (1993). The optical images, broad-band $R$ and narrow-band $\mathrm{H} \alpha$, are from Larsen \& Richtler (1999) and Lundgren et al. (2005). All images were smoothed to a common resolution of $23^{\prime \prime}(\approx 500 \mathrm{pc})$. The nuclear region was screened with a diameter of $1^{\prime}$, since we expect the nuclear area to behave differently from the outer bulge, spiral arm and interarm regions. We assume a relationship between the intensities $X$ and $Y$ at two wavelengths of the form:

$Y=10^{\text {Const }} \cdot X^{\text {Exp }}$.

The exponents, with the associated errors, as well as the Pearson's correlation coefficients and Student-t values, are given in Table 5. Representative plots are presented in Fig. 14.

The correlation between $L W 2$ and $L W 3$ is extremely good; the ratio $L W 2 / L W 3$ shows little variation within the disk (see Sect. 7.2 and the radial profiles in Fig. 11). The correlations of $L W 2$ and $L W 3$ with the radio continuum and with the $R$ band are likewise good, with a Pearson's correlation coefficient of $\sim 0.88$, closely followed by that of $\mathrm{H} \alpha$, having correlation coefficients of 0.80 to 0.84 . The total radio continuum emission is better correlated with the MIR emission than with 
Table 5. Correlations $X \longleftrightarrow Y$ between the logarithms of the intensities of various emissions in M 83 at $23^{\prime \prime}$ resolution, computed between 0.5 and $5^{\prime}$ distance from the center in the galaxy plane. The first value tabulated is the slope of the regression line (the exponent in Eq. (1)) and its error, followed by the Pearson's correlation coefficient $r$, its error, and the $t$ value of Student's t-test (see text for details).

\begin{tabular}{|c|c|c|c|c|c|c|c|c|}
\hline$X \backslash Y$ & $15 \mu \mathrm{m}$ & Radio & Thermal & Nonthermal & $R$ band & $\mathrm{H} \alpha$ & $\mathrm{CO}(J=1-0)$ & $\mathrm{HI}$ \\
\hline \multirow[t]{5}{*}{$7 \mu \mathrm{m}$} & 1.05 & 0.86 & $\approx 0.8$ & 0.8 & 0.89 & 1.26 & 0.9 & 0.65 \\
\hline & \pm 0.03 & \pm 0.12 & - & \pm 0.3 & \pm 0.06 & \pm 0.05 & \pm 0.2 & \pm 0.14 \\
\hline & 0.978 & 0.89 & 0.74 & 0.78 & 0.89 & 0.84 & 0.70 & 0.43 \\
\hline & \pm 0.004 & \pm 0.02 & \pm 0.07 & \pm 0.04 & \pm 0.02 & \pm 0.03 & \pm 0.07 & \pm 0.07 \\
\hline & 48 & 21 & 7 & 13 & 21 & 15 & 7 & 5 \\
\hline \multirow[t]{5}{*}{$15 \mu \mathrm{m}$} & & 0.88 & $\approx 0.8$ & 0.8 & 0.89 & 1.25 & 0.9 & 0.7 \\
\hline & & \pm 0.14 & - & \pm 0.3 & \pm 0.09 & \pm 0.06 & \pm 0.2 & \pm 0.2 \\
\hline & & 0.88 & 0.73 & 0.82 & 0.87 & 0.80 & 0.72 & 0.26 \\
\hline & & \pm 0.02 & \pm 0.07 & \pm 0.03 & \pm 0.03 & \pm 0.04 & \pm 0.06 & \pm 0.09 \\
\hline & & 18 & 7 & 14 & 18 & 13 & 8 & 3 \\
\hline \multirow[t]{5}{*}{ Radio } & & & 1.0 & 0.9 & 1.08 & 1.36 & 1.0 & 0.8 \\
\hline & & & \pm 0.8 & \pm 0.3 & \pm 0.08 & \pm 0.13 & \pm 0.5 & \pm 0.2 \\
\hline & & & 0.79 & 0.90 & 0.85 & 0.68 & 0.77 & 0.53 \\
\hline & & & \pm 0.06 & \pm 0.02 & \pm 0.02 & \pm 0.06 & \pm 0.05 & \pm 0.06 \\
\hline & & & 8 & 21 & 19 & 9 & 9 & 7 \\
\hline \multirow[t]{5}{*}{ Thermal } & & & & 1.1 & 1.7 & 1.5 & 1.0 & 0.9 \\
\hline & & & & \pm 0.8 & \pm 0.4 & \pm 0.4 & \pm 0.7 & \pm 0.2 \\
\hline & & & & 0.58 & 0.57 & 0.70 & 0.60 & 0.50 \\
\hline & & & & \pm 0.11 & \pm 0.10 & \pm 0.08 & \pm 0.12 & \pm 0.12 \\
\hline & & & & 4 & 5 & 6 & 4 & 4 \\
\hline \multirow[t]{5}{*}{ Nonthermal } & & & & & 1.13 & 1.5 & 1.1 & $\approx 0.9$ \\
\hline & & & & & \pm 0.14 & \pm 0.2 & \pm 0.6 & - \\
\hline & & & & & 0.79 & 0.53 & 0.69 & $<0.1$ \\
\hline & & & & & \pm 0.04 & \pm 0.08 & \pm 0.07 & - \\
\hline & & & & & 13 & 6 & 7 & $<1$ \\
\hline \multirow[t]{5}{*}{$R$ band } & & & & & & 1.3 & $\approx 1.0$ & $\approx 0.5$ \\
\hline & & & & & & \pm 0.2 & - & - \\
\hline & & & & & & 0.60 & 0.66 & 0.35 \\
\hline & & & & & & \pm 0.07 & \pm 0.08 & \pm 0.07 \\
\hline & & & & & & 7 & 7 & 4 \\
\hline \multirow[t]{5}{*}{$\mathrm{H} \alpha$} & & & & & & & 0.7 & 0.5 \\
\hline & & & & & & & \pm 0.6 & \pm 0.2 \\
\hline & & & & & & & 0.52 & 0.32 \\
\hline & & & & & & & \pm 0.10 & \pm 0.09 \\
\hline & & & & & & & 4 & 3 \\
\hline \multirow[t]{5}{*}{$\mathrm{CO}(J=1-0)$} & & & & & & & & $\approx-0.6$ \\
\hline & & & & & & & & - \\
\hline & & & & & & & & $<0.1$ \\
\hline & & & & & & & & - \\
\hline & & & & & & & & $<1$ \\
\hline
\end{tabular}

each of the individual thermal and non-thermal radio components. The $\mathrm{CO}$ emission does not follow the MIR emission well (correlation coefficent of 0.7 ). The $\mathrm{H} \alpha$ and the radio thermal emission are not well correlated, probably an effect due to optical extinction. The $\mathrm{H} \alpha$ and the $R$ band are also not wellcorrelated which is evident in Fig. 11.
H I shows no significant correlation with any other tracer. All these results agree with those found in NGC 6946 (Frick et al. 2001; Walsh et al. 2002).

We also studied correlations between the images available at $12^{\prime \prime}(260 \mathrm{pc})$ resolution which gave very similar results. For example, the correlation coefficient of the $L W 2-L W 3$ 

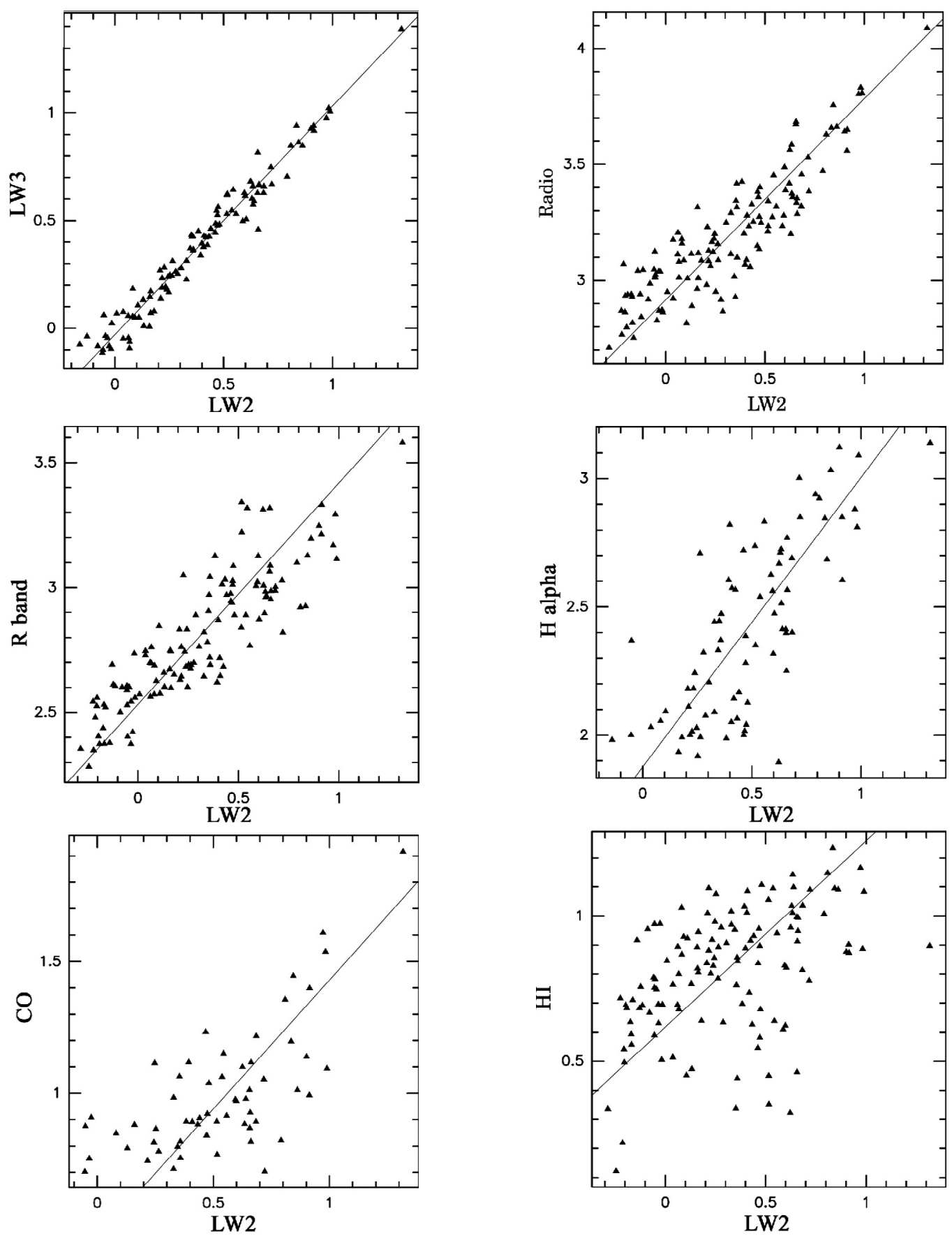

Fig. 14. Correlations for different tracers of the interstellar medium in M 83 excluding the nuclear region $\left(0.5^{\prime} \leq R \leq 5^{\prime}\right)$. Images at a resolution of $23^{\prime \prime}$ were used. Points in the diagrams represent independent pixels separated by two beam widths and having signal-to-noise ratios of $\geq 5$. We show the correlations between the logarithms of intensities (in arbitrary units) in MIR filters $L W 2$ (centered at $\lambda 6.75 \mu \mathrm{m})$ and $L W 3(\lambda 15 \mu \mathrm{m}$ ), between $L W 2$ and $\lambda 6 \mathrm{~cm}$ radio continuum, $L W 2$ and optical $R$ band, $L W 2$ and $\mathrm{H} \alpha, L W 2$ and $\mathrm{CO}$, and between $L W 2$ and $\mathrm{HI}$.

correlation was 0.95 and that of the $L W 3$-radio continuum correlation was 0.84 .

\subsection{The mid-infrared LW2 $(\lambda 6.75 \mu \mathrm{m})-$ LW3 $(\lambda 15.0 \mu \mathrm{m})$ correlation}

Figures 1 and 14 (top left panel) show an excellent correlation between the two MIR broad-band filters. The correlation coefficient is close to one $(0.978 \pm 0.004)$ and the exponent $1.05 \pm 0.03$, arguing for a nearly linear relationship. In NGC 6946 (Walsh et al. 2002), the correlation coefficient and the exponent are almost identical to those in M 83. The $L W 2 / L W 3$ ratios (Table 3) vary within M 83 from 0.6 to 1.75 . Since we screened the nuclear and inner bulge region and required a signal-to-noise ratio $\geqq 5$, thus excluding a part of the interarm regions, we apparently pick up ratios of 1.1 for the outer bulge and 0.8 for a region on the spiral arm (with errors of $\sim 20 \%-30 \%$ ). 
The overall $L W 2 / L W 3$ ratio $\sim 1$ is regarded to be typical for spiral galaxies without active nuclei (Roussel et al. 2001a,b; see Sect. 3.5). Applying our findings to the case of spatially unresolved galaxies, $L W 2 / L W 3$ ratios $\sim 1$ for the total galaxy do not exclude the existence of bursts of star formation in particular regions like the nucleus or the inner spiral arms, where $L W 2 / L W 3<1$ (see also Roussel et al. 2001c).

\subsection{The mid-infrared - radio correlation}

The correlation between the integrated radio continuum and far-infrared (FIR) fluxes of galaxies is one of the closest in astrophysics and has been discussed over many years (e.g. Helou \& Bicay 1993; Niklas \& Beck 1997; Hoernes et al. 1998). The mid-infrared - radio correlation within M 83 is also very tight (Figs. 1, 7, 14). The correlation coefficients between the $L W 2$ and $L W 3$ MIR bands and the radio intensity are $0.89 \pm 0.02$ and $0.88 \pm 0.02$, respectively (Table 5 ). The slopes of $0.86 \pm 0.12$ and $0.88 \pm 0.14$ are similar to that of the far-infrared - radio correlation (Niklas \& Beck 1997; Hoernes et al. 1998) and indicate that the same physical process is involved. The warm dust consists of PAHs and grains of different sizes, emitting in the mid- and far-infrared. Neither the individual thermal nor non-thermal radio components are better correlated with MIR than the total radio emission. The MIR emission, similar to the FIR emission, probably consists of two components, a diffuse one (like that of the non-thermal radio emission) and a clumpy one concentrated around star-forming regions (like that of the thermal radio emission).

The explanation of the correlation may be threefold:

a) thermal emission;

b) cosmic-ray production in star-formation regions;

c) magnetic fields.

a) If the radio continuum intensity in M 83 is dominated by free-free thermal emission, the correlation could be caused by the action of star formation which heats the dust and ionizes the gas. However, thermal radio emission originates in the ionized gas phase, a phase which primarily provides the dust continuum component of the $L W 3$ emission and is also responsible for the excitation of the surrounding PAHs dominating the $L W 2$ emission. Furthermore, our separation of thermal and non-thermal radio emission of M 83, with the help of the spectral index, reveals a dominance of non-thermal emission at all radii. Thermal emission may dominate locally in star-forming regions, but non-thermal processes are essential in order to understand the correlation.

b) Star-forming regions are locations of type II, Ib and Ic supernovae, and the shock fronts of their remnants are probably cosmic-ray accelerators. If the magnetic field strength were constant, the radio continuum image would reflect the distribution of cosmic-ray electrons originating in star-forming regions. The diffusion speed of cosmic rays is limited to the Alfvén velocity $\left(\simeq 100 \mathrm{~km} \mathrm{~s}^{-1}\right.$ in the hot interstellar medium) so that the particles propagate $\simeq 1 \mathrm{kpc}$ within their lifetime of $\simeq 10^{7}$ yr. Under the given assumptions the radio continuum map should look like a smoothed image of the star-forming regions $(\mathrm{H} \alpha)$, which indeed is the case. Similarly, the MIR image traces the dust in and around the star-forming regions. A detailed analysis of this correlation "scale by scale" with help of wavelets is underway (Beck et al. 2005).

c) However, a constant magnetic field strength in the disk of M 83 is physically unreasonable. Even small variations in field strength lead to strong fluctuations in synchrotron intensity (see Sect. 4). In this case any correlation with radio continuum emission needs to consider magnetic fields.

The lack of large-scale correlations of radio continuum with neutral H I gas or with hot X-ray emitting gas from the disk (Ehle et al. 1998) indicates that the warm neutral gas as well as the hot ionized gas components are of little importance for the distribution of magnetic fields.

We propose that the interstellar magnetic fields are connected to the gas clouds in which the dust is embedded and they are probably anchored in their photoionized shells. This provides a relation between the number density $N$ of gas clouds and field strength $B$ of $B^{2} \propto N$. A similar relation is obtained through equipartition between the energy densities of turbulent cloud motion and magnetic fields, as e.g. observed in NGC 6946 (Beck 2004). The physical mechanism and time scale of this coupling has to be investigated.

Our result also bears important consequences for the generation and dynamics of magnetic fields. If the fields are anchored in gas clouds, turbulent motions of gas clouds may cause the " $\alpha$-effect" necessary for dynamo action (Beck et al. 1996).

Elbaz et al. (2002) have demonstrated the existence of the global MIR-radio correlation for galaxies up to $z=1$, while Appleton et al. (2004) have found this correlation to remain out to $z=2$. Thus, globally for galaxies in the local universe and within individual galaxies, as we see here, as well as for cosmologically significant galaxies, this correlation remains a fundamentally important physical process.

\subsection{Comparison between mid-infrared and $\mathrm{H} \alpha$}

In our Galaxy, the PAH emission seems to be closely associated with photodissociation shells and the surfaces of molecular clouds in the vicinity of $\mathrm{H}$ II regions or exciting hot stars, while the VSG continuum peaks inside H II regions (Siebenmorgen \& Krügel 1992; Cesarsky et al. 1996; Verstraete et al. 1996). Both regions are linked to star formation, which is also traced by $\mathrm{H} \alpha$ emission. From this, we would expect a correlation between the MIR and $\mathrm{H} \alpha$ emission (smoothed to the same resolution). For M 83 we find a correlation coefficient of $\simeq 0.84$ and $f_{\mathrm{H} \alpha} \sim f_{\mathrm{MIR}}^{1.3}$ (Table 5). According to Fig. 8, the MIR emission is more diffuse than $\mathrm{H} \alpha$ which is clumpy and concentrated toward the youngest star-forming regions. This also explains the tight correlation between the MIR and optical $R$ band emissions.

While the radial profiles of $\mathrm{H} \alpha$ and the $L W 2$ and $L W 3$ appear to follow a similar pattern, there are significant relative spatial differences near $2^{\prime}$, where the peak is more pronounced in $\mathrm{H} \alpha$ than in the MIR. Differences are also apparent in the outer disk region, $r>4^{\prime}$ (Fig. 11). These effects result in a slope of the regression exponent being 1.3 (Fig. 14). This exponent predicts, in principle, the detection of 
decreasing MIR emission with respect to the $\mathrm{H} \alpha$ emission in the case of increasing radiation fields. Given the large scatter of the individual values, this result should be taken with care.

Roussel et al. (2001b) report on the relationship between star-formation rates and mid-infrared emission in galactic disks for a sample of 69 galaxies. Excluding the nuclear regions, they find, within their errors, a linear relationship between the total MIR and $\mathrm{H} \alpha$ emission in disks. The question arises whether, on a much larger scale than the investigated one $\left(12^{\prime \prime} \widehat{\equiv} 260 \mathrm{pc}\right)$, the MIR and $\mathrm{H} \alpha$ emission correlate linearly. To check this, we made pixel by pixel comparisons, requiring the same signal to noise ratio, with artificially-reduced resolution. Reducing the resolution by a factor of 2,4 or 8 still leads to a large scatter. With a resolution of $96^{\prime \prime} \widehat{\equiv} 2 \mathrm{kpc}$ we find good correlations between any tracers except $\mathrm{H} \mathrm{I}$ (correlation coefficients of $0.98 \pm 0.01$, but Student-t values of only $\simeq 10$ ). This reflects the obvious fact that an extended disk exists in most spectral ranges (see Fig. 1). Thus correlation analysis of low-resolution data can be misleading. A scale separation is required, as demonstrated for NGC 6946 by Frick et al. (2001).

\subsection{Classification of M83 as a starburst galaxy with the help of its MIR colours}

We calculated the $L W 3 / L W 2$ and $L W 2 / L W 4$ ratios for the five regions presented in Sect. 3.2 and Fig. 3. The ratios are plotted in Fig. 15. The ratios can be used to build a classification diagram (Laurent et al. 2000), in which the MIR emission from AGNs, photo-dissociation regions (PDR) and H II regions are separated. The expectations for pure AGN, PDR and H II regions and the results of Laurent et al. (2000) for the AGN-dominated galaxy NGC 1068 and the starburst dominated galaxy M 82 are indicated. Our measurements for the nucleus, bulge and inner disk of M 83 are in the vicinity of the M 82 measurements arguing for a high rate of star formation in these regions. The regions 4 and 5 are slightly offset, which suggests a different physical state. One should, however, keep in mind that the ratios for the faint regions 4 and 5 are affected by larger errors $(\sim 30 \%)$.

\section{Summary}

M 83 was observed with the ISOCAM in the $4 \mu \mathrm{m}$ to $18 \mu \mathrm{m}$ band.

- The $L W 2$ and $L W 3$ broad-band filter observations covered the entire optical extent $\left(d_{25}=11.5 \widehat{=} 15 \mathrm{kpc}\right.$ at $D=4.5 \mathrm{Mpc}$ assumed distance) of the galaxy. Both filters show bright emission from the nuclear and bulge regions, star-formation regions at the tips of the bar and the inner spiral arms. Emission from the outer spiral arms and interarm regions is discernible with low intensity only. For $L W 2$ ( $\lambda 5 \mu \mathrm{m}$ to $8.50 \mu \mathrm{m})$ and $L W 3(\lambda 12 \mu \mathrm{m}$ to $18 \mu \mathrm{m})$, we measure an integral flux for M 83 of $20 \mathrm{Jy}\left(1.6 \times 10^{43} \mathrm{erg} \mathrm{s}^{-1}\right)$ and $21 \mathrm{Jy}\left(6.6 \times 10^{42} \mathrm{erg} \mathrm{s}^{-1}\right)$, respectively. The central $3^{\prime} \times 3^{\prime}$ $(\widehat{=} 4 \mathrm{kpc} \times 4 \mathrm{kpc})$ region contributes $28 \%$ and $32 \%$ to the total mid-infrared emission in the case of $L W 2$ and $L W 3$, respectively.

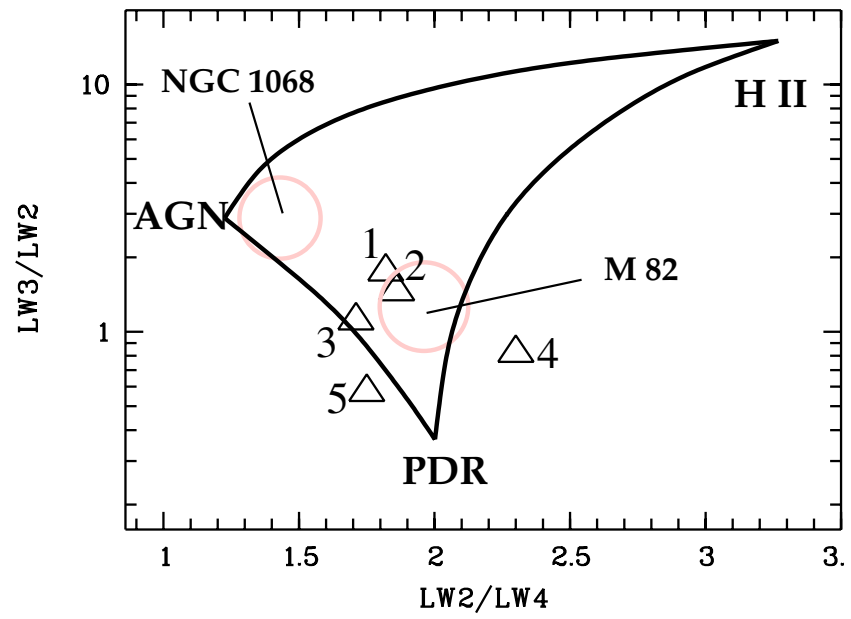

Fig. 15. Classification diagram according to Laurent et al. (2000). The positions of the extracted regions $1-5$ of Sect. 3.2 are marked with triangles in this diagram.

- Strip maps, centered on the nucleus of M83, were taken in seven narrow-band filters with scan directions from the north-east to the south-west. The $(14.0 \mu \mathrm{m}-16.0 \mu \mathrm{m}) /(7.0 \mu \mathrm{m}-8.5 \mu \mathrm{m})$ ratio is found to be a good tracer of star-formation regions in the galaxy.

- The central $3 ! 2 \times 3.2(\widehat{=} 4 \mathrm{kpc} \times 4 \mathrm{kpc})$ region of $\mathrm{M} 83$ was also observed in the spectral imaging mode. This allowed us to extract $\lambda 4 \mu \mathrm{m}$ to $18 \mu \mathrm{m}$ spectra for 5 different regions: the center, the inner and outer bulge, a midinfrared emitting on-arm region close to the south-western tip of the bar, as well as an averaged interarm region spectrum. PAH bands are identified in all regions at $\lambda 6.2 \mu \mathrm{m}$, $7.7 \mu \mathrm{m}, 8.6 \mu \mathrm{m}$ and $11.3 \mu \mathrm{m}$, as well as a blended [NeII] and $\mathrm{PAH}$ feature at $\simeq 12.8 \mu \mathrm{m}$.

- Summed over the total ISOCAM MIR wavelength range of $\lambda 4$ to $18 \mu \mathrm{m}$, the PAHs dominate in energy over the small grain continuum emission. While dust continuum contributions make up $40 \%$ of the total flux in the central region, they become almost negligible in the interarm regions $(\lesssim 10 \%)$. The contribution of atomic lines are small in general, the brightest line being the [NeII] line towards the center. The ratio $L W 2 / L W 3$ varies from $\simeq 0.6$ (nucleus) to $\simeq 1.8$ (interarm regions).

- From our spectral imaging mode, we tested the PAH versus continuum distribution for the broad-band filters $L W 2$ and $L W 3$. A great number of galaxies have only been observed in these filter bands which were thought to trace mainly the PAHs ( $L W 2)$ and dust continuum (LW3). For $L W 2$ we find that $\mathrm{PAH}$ features indeed dominate the emission in the case of all regions. For the $L W 3$ band, the continuum emission dominates in the case of the nucleus, bulge and spiral arms. For interarm or more diffuse regions, however, PAH features are found to be the main source of the $L W 3$ emission.

New radio continuum (a combined $\lambda 6 \mathrm{~cm}$ VLA/Effelsberg map) and $\mathrm{H} \alpha$ data, which were used herein for a detailed quantitative analysis, are presented. Both wavelengths show similar morphological features as the mid-infrared emission does. 
Further tracers of the interstellar medium, $\mathrm{CO}(J=1-0), \mathrm{HI}$, diffuse $\mathrm{X}$-rays, as well as $R$ band were compared to the midinfrared images.

- The radial profiles of the MIR, $\mathrm{H} \alpha$, hard X-ray and thermal radio continuum intensities in the range $2^{\prime}-4^{\prime}$ have the same scale length of $1.3 \pm 0.1(1.7 \pm 0.1 \mathrm{kpc})$ and steepen beyond $5 \mathrm{kpc}$ radius. The radial declines of the total radio continuum, $\mathrm{CO}$ and broad-band optical emission are significantly flatter (scale lengths $1.7-2 ! 2$ ).

- The morphology of the radio continuum and, to a large degree, $\mathrm{H} \alpha$ resembles that of the mid-infrared. Correlation studies between both two mid-infrared broad-bands and the radio continuum intensities $I$ at $23^{\prime \prime}$ resolution show a nearly linear dependence $\left(I_{\text {radio }}=\right.$ const $\left.\cdot I_{L W 2}^{0.86 \pm 0.12}\right)$ with a correlation coefficient of $0.89 \pm 0.02$. In the case of $\mathrm{H} \alpha$ we obtain $I_{\mathrm{H} \alpha}=$ const $\cdot I_{L W 2}^{1.26 \pm 0.05}$ with a correlation coefficient of $0.84 \pm 0.03$.

- The close relationship between mid-infrared and $\lambda 6 \mathrm{~cm}$ radio continuum is discussed in the framework of different scenarios. A possible implication of our findings is that the interstellar magnetic fields are anchored in the photoionized shells of gas clouds. This result would have important consequences for the generation and dynamics of magnetic fields, e.g., the " $\alpha$-effect" necessary for dynamo action may be driven by turbulent motions of gas clouds.

Acknowledgements. We wish to thank N. Neininger for help with the radio observations, Ch. Nieten for providing and explaining his correlation software, and T. Handa, G. Petitpas, R. Rand and R. Tilanus for kindly providing their $\mathrm{CO}(J=1-0), \mathrm{CO}(J=4-3), \mathrm{CO}(J=1-0$, $\mathrm{S}$ arm) and HI images. We thank H. Roussel who provided the preanalyzed ISOCAM CVF data cube. We thank E. Krügel for his insightful reading of the paper. We appreciate the careful reading of the manuscript by our referee and comments that helped improve the clarity of the presentation. Part of the MIR analysis was carried out with non-standard CIA software written by P. Chanial, H. Roussel and D. Reynaud. O. Laurent provided the source codes for the spectral analysis of the CVF data. Our work made use of the archival data base at the European Southern Observatory (ESO) and the NASA Extragalactic Database (NED).

\section{References}

Adamson, A. J., Adams, D. J., \& Warwick, R. S. 1987, MNRAS, 224, 367

Allamandola, L. J., Tielens, A. G. G. M., \& Barker, J. R. 1989, ApJS, 71,733

Allamandola, L. J., Hudgins, D. M., \& Sandford, S. A. 1999, ApJ, 511, L115

Appleton, P. N., Fadda, D., Marleau, F. R., et al. 2004, ApJ, 154, 147

Arendt, R. G., Dwek, E., \& Mosley, S. H. 1999, ApJ, 521, 234

Athanassoula, E. 1992, MNRAS, 259, 345

Athey, A., Bregman, J., Bregman, J., Temi, P., \& Sauvage, M. 2002, ApJ, 571, 272

Beck, R. 2002, in Disks of Galaxies: Kinematics, Dynamics and Pertubations, ed. E. Athanassoula et al., ASP Conf. Ser., 275, 331

Beck, R. 2004, in How Does the Galaxy Work?, ed. E. J. Alfaro et al. (Dordrecht: Kluwer), 277

Beck, R., \& Hoernes, P. 1996, Nature, 379, 47
Beck, R., Brandenburg, A., Moss, D., Shukurov, A., \& Sokoloff, D. 1996, ARA\&A, 34, 155

Beck, R., Ehle, M., Shoutenkov, V., Shukurov, A., \& Sokoloff, D. 1999, Nature, 397, 324

Beck, R., Ehle, M., Frick, P., et al. 2005, in prep.

Bland, J., \& Tully, B. 1988, Nature, 334, 43

Boselli, A., Gavazzi, G., \& Sanvito, G. 2003, A\&A, 402, 37

Boulanger, F., Boissel, P., Cesarsky, D., \& Ryter, C. 1998, A\&A, 339, 194

Bresolin, F., \& Kennicutt, R. C. 2002, ApJ, 572, 838

Cesarsky, C. J., Abergel, A., Agnese, P., Altieri, B., \& Augueres, J. L. 1996a, A\&A, 315, 32

Cesarsky, D., Lequeux, J., Abergel, A., et al. 1996b, A\&A, 315, L309

Chan, K.-W., Roellig, T. L., Onaka, T., et al. 2001, ApJ, 546, 273

Chanial, P., \& Gastaud, R. 2000 (software available on email request via cir@discovery.saclay.cea.fr)

Coulais, A., \& Abergel, A. 2000, A\&AS, 141, 533

Crosthwaite, L. P., Turner, J. L., Buchholz, L., et al. 2002, AJ, 123, 1892

Dale, D. A., Silbermann, N. A., Helou, G., et al. 2000, AJ, 120, 583

Désert, F.-X., Boulanger, F., \& Puget, J. L. 1990, A\&A, 237, 215

Deutsch, E. W., \& Allen, R. J. 1993, AJ, 106, 1812

Dickey, J. M., \& Lockman, F. J. 1990, ARA\&A, 28, 215

Dumke, M., Thuma, G., Walsh, W., et al. 2005, A\&A, in prep.

Ehle, M., Pietsch, W., Beck, R., \& Klein, U. 1998, A\&A, 329, 39

Elbaz, D., Cesarsky, C. J., Chanial, P., et al. 2002, A\&A, 384, 848

Elmegreen, D. M., Chromey, F. R., \& Warren, A. R. 1998, AJ, 116, 2834

Förster-Schreiber, N. M., Sauvage, M., Charmandaris, V., et al. 2003, A\&A, 399, 833

Frick, P., Beck, R., Berkhuijsen, E. M., \& Patrickeyev, I. 2001, MNRAS, 327, 1145

Gallais, P., Rouan, D., Lacombe, F., Tiphéne, D., \& Vauglin, I. 1991, A\&A, 243, 309

Galliano, F. 2004, Ph.D. Dissertation, University of Paris

Genzel, R., Lutz, D., Sturm, E., et al. 1998, ApJ, 498, 579

Genzel, R., \& Cesarsky, C. J. 2000, ARA\&A, 38, 761

Handa, T., Nakai, N., Sofue, Y., Hayashi, M., \& Fujimoto, M. 1990, PASJ, 42, 1

Handa, T., Ishizuki, S., \& Kawabe, R. 1994, in Astronomy with Millimeter and Submillimeter Wave Interferometry, ASP Conf. Ser., 59, 341

Helou, G., \& Bicay, M. D. 1993, ApJ, 415, 93

Helou, G., Malhotra, S., Beichman, C., et al. 1996, A\&A, 315, L157

Henning, T., Klein, R., Launhardt, R., Lemke, D., \& Pfau, W. 1998, A\&A, 332, 1035

Hoernes, P., Berkhuijsen, E. M., \& Xu, C. 1998, A\&A, 334, 57

Huchtmeier, W. K., \& Bohnenstengel, H.-D. 1981, A\&A, 100, 72

Hunt, L. K., Giovanardi, C., \& Helou, G. 2002, A\&A, 394, 873

Immler, S., Vogler, A., Ehle, M., \& Pietsch, W. 1999, A\&A, 352, 415

Jourdain de Muizon, M., d'Hendecourt, L. B., \& Geballe, T. R. 1990, A\&A, 227, 526

Kessler, M., Steintz, J., Anderegg, M., et al. 1996, A\&A, 315, L27

Krügel, E. 2003. The Physics of Interstellar Dust (Bristol: Institute of Physics Publishing), 271

Lagage, P. O., Claret, A., Ballet, J., et al. 1996, A\&A, 315, L273

Larsen, S. S., \& Richtler, T. 1999, A\&A, 345, 59

Laurent, O., Mirabel, I. F., Charmandaris, V., et al. 2000, A\&A, 359, 887

Leech, K. J., Metcalfe, L., \& Altiere, B. 2001, MNRAS, 328, 1125

Lehnert, M. D., Heckman, T. M., \& Weaver, K. A. 1999, ApJ, 523, 575

Lemke, D., Mattila, K., \& Lehtinen, K. 1998, A\&A, 331, 742 
Lord, S. D., \& Kenney, J. D. P. 1991, ApJ, 381, 130

Lundgren, A. A., Wiklind, T., Olofsson, H., \& Rydbeck, G. 2004a, A\&A, 413, 505

Lundgren, A. A., Wiklind, T., Olofsson, H., \& Rydbeck, G. 2004b, A\&A, 422, 865

Lundgren, A. A., Olofsson, H., \& Wiklind, T., 2005, A\&A, submitted

Lutz, D., Sturm, E., Genzel, R., et al. 2003, A\&A, 409, 867

Madden, S. C., Galliano, F., Jones, A., \& Sauvage, M. 2005, A\&A, submitted

Mattila, K., Lemke, D., Haikala, L. K., et al. 1996, A\&A, 315, L353

Neininger, N., Klein, U., Beck, R., \& Wielebinski, R. 1991, Nature, 352,781

Neininger, N., Beck, R., Sukumar, S., \& Allen, R. J. 1993, A\&A, 274, 687

Nieten, C. 2001, Ph.D. Thesis, University of Bonn

Niklas, S., \& Beck, R. 1997, A\&A, 320, 54

Ohashi, T., Makishima, K., Tsuru, T., et al. 1990, ApJ, 365, 180

Okada, K., Mitsuda, K., \& Dotani, T. 1997, PASJ, 49, 653

Petitpas, G. R., \& Wilson, C. D. 1998, ApJ, 503, 219

Puget, J. L., \& Leger, A. 1984, A\&A, 137, L5

Rand, R. J., Lord, S. D., \& Higdon, J. L. 1999, ApJ, 513, 720

Rigopoulou, D., Kunze, D., Lutz, D., Genzel, R., \& Moorwood, A. F. M. 2002, A\&A, 389, 374

Roussel, H., Sauvage, M., Bosma, A., et al. 2001a, A\&A, 369, 473

Roussel, H., Sauvage, M., Vigroux, L., et al. 2001b, A\&A, 372, 406

Roussel, H., Sauvage, M., Vigroux, L., et al. 2001c, A\&A, 372, 427

Ryder, S. D., Hungerford, A., Dopita, M. A., et al. 1995, in The Opacity of Spiral Disks, ed. J. I. Davies, \& D. Burstein (Dordrecht: Kluwer), 359

Ryder, S. D., \& Dopita, M. A. 1994, ApJ, 430, 142
Sakamoto, K., Matsushita, S., Peck, A. B., Wiedner, M. C., \& Iono, D. 2004, ApJ, 616, L59

Schlegel, D. J., Finkbeiner, D. P., \& Davis, M. 1998, ApJ, 500, 525

Schulz, H., \& Wegner, G. 1992, A\&A, 266, 167

Schutte, W. A., Tielens, A. G. G. M., \& Allamandola, L. J. 1993, ApJ, 415,397

Sellgren, K., Luan, L., \& Werner, M. W. 1990, ApJ, 359, 384

Siebenmorgen, R., \& Krügel, E. 1992, A\&A, 259, 614

Sofue, Y., \& Wakamatsu, T. 1994, AJ, 107, 1018

Soria, R., \& Wu, K. 2002, A\&A, 384, 99

Soria, R., \& Wu, K. 2003, A\&A, 410, 53

Sturm, E., Lutz, D., Tran, D., et al. 2000, A\&A, 358, 481

Sturm, E., Lutz, D., Verma, A., et al. 2002, A\&A, 393, 821

Sukumar, S., \& Allen, R. J. 1989, Nature, 340, 537

Thatte, N., Tecza, M., \& Genzel, R. 2000, A\&A, 364, L47

Thim, F., Tammann, G. A., Saha, A., et al. 2003, ApJ, 590, 256

Tilanus, R. P. J., \& Allen, R. J. 1993, A\&A, 274, 707

Trinchieri, G., Fabbiano, G., \& Palumbo, G. G. C. 1985, ApJ, 290, 96

Uchida, K. I., Sellgren, K., Werner, M. W., \& Houdashelt, M. L. 2000, ApJ, 530, 817

Verma, A., Lutz, D., Sturm, E., et al. 2003, A\&A, 403, 829

Vermeij, R., Peeters, E., Tielens, A. G. G. M., \& van der Hulst, J. M. 2002, A\&A, 382, 1042

Verstraete, L., Puget, J. L., Falgarone, E., et al. 1996, A\&A, 315, L337

Verstraete, L., Pech, C., Moutou, C., et al. 2001, A\&A, 372, 981

Vigroux, L., Charmandaris, V., Gallais, P., et al. 1999, in The Universe as Seen by ISO, ed. P. Cox, \& M. F. Kessler, ESA-SP, 427, 805

Walsh, W., Beck, R., Thuma, G., et al. 2002, A\&A, 388, 7

Xilouris, E. M., Madden, S. C., Galliano, F., Vigroux, L., \& Sauvage, M. 2004, A\&A, 416, 41 